\title{
New magnetic flux flows with Heisenberg ferromagnetic spin of optical quasi velocity magnetic flows with flux density
}

\author{
T. Körpinar ${ }^{a}$, R. Cem Demirkol ${ }^{b}$, Z. Körpinar ${ }^{c}$, and V. Asil ${ }^{d}$ \\ ${ }^{a, b}$ Muş Alparslan University, Department of Mathematics, 49250, Muş, Turkey, \\ ${ }^{c}$ Muş Alparslan University, Department of Administration, 49250, Muş, Turkey, \\ ${ }^{d}$ Firat University, Department of Mathematics, 23100, Elazı $\breve{g}$, Turkey.
}

Received 5 October 2020; accepted 16 December 2020

In this article, we first offer the approach of quasi magnetic Lorentz flux of quasi velocity magnetic flows of particles by the quasi frame in 3D space. We then obtain new optical conditions of quasi magnetic Lorentz flux by using directional quasi fields. Moreover, we determine the quasi magnetic Lorentz flux for quasi vector fields. Additionally, we give new constructions for quasi curvatures of quasi velocity magnetic flows by considering Heisenberg ferromagnetic spin. Finally, the magnetic flux surface is demonstrated on a static and uniform magnetic surface by using the analytical and numerical results.

Keywords: Quasi directional frame; flows; Heisenberg ferromagnetic spin; geometric magnetic flux density.

PACS: 04.20.-q; 03.50.De; 02.40.-k.

DOI: https://doi.org/10.31349/RevMexFis.67.378

\section{Introduction}

The magnetic flux density is an important theoretical model for quantum physics, quantum magnetics, and quantum optics. Magnetic flux density determines the optical solitons for magnetic flux and electric flux density across the symmetry. The flux density has been studied by numerous scientists from different viewpoints. Lorentz flux is an important subclass of the flux density and it is more related to the theory of optical propagation. One can also find a correlation between optical and magnetic soliton solutions of different models by considering the flux density.

An ideal optical fiber has perfect circular symmetry. The polarizations are completely degenerate. Perturbations and imperfections during the fabrication process may introduce anisotropies, which are mostly of a linear or Cartesian type. Bending and stretching optical fibers does also determine linear birefringence. Rotational effects of polarization are difficult to produce since their production process has not been fully comprehended for many cases.

In recent times, the advancement of glowing lasers and the utilization of optical fiber mechanics have increased the importance of flow propagation by curled fluid flows and space-curved. Exclusively torque forces of the geometric phase of isolated light anholonomy with some optical fibers have been investigated by numerous researchers. For example, Smith [1] examined that a torque of the divergence of light is generated along with the monochromatic optical fiber bundle thanks to the magnetic particle's flows in a certain transformer. Another preliminary geometric effect of the torque of magnetic divergence of light propagating in a magnetic optical fiber detecting a magnetic trajectory was presented by [2]. Ross improved a totally geometric system to investigate the coiled optical fiber with a fixed-torsion and investigated its effects with several measurements. Tomita and Chiao [3] summarized the previous review of Ross for more general fiber shapes. Also, Chiao and $\mathrm{Wu}$ [4] obtained an important theoretical phase as a result of geometric phase torque. Haldane defined the geometric rotation of the polarization angle in an ideal cylindrical optical fiber without birefringence for arbitrary fiber paths in terms of the image of the path in the tangent vector space [5]. Apart from previous researches, we proposed some new approaches to compute the electromagnetic phase with an antiferromagnetic chain [6$10]$.

The geometric phase investigation along the optical fiber investigation is mostly conducted by observing the action of electromagnetic particles and their features. Some nonlinear evolution structures are frequently encountered particularly in genuine-state physics, chemical physics, plasma physics, optical physics, fluid mechanics, etc. Even though these equations have been heavily used in many structures, it requires very hard work to obtain the explicit solutions of approximate systems. Thus, there exists no global or unified approach to demonstrate the exact solutions of all nonlinear transformation systems [11-23].

The aim of the present paper is to introduce a new geometric interpretation of the notion of the Heisenberg ferromagnetic spin for quasi flows of magnetic particles with the quasi-frame in the space. Eventually, we obtain new optical conditions of quasi magnetic Lorentz flux by using directional quasi fields. Moreover, we determine the quasi magnetic Lorentz flux for quasi vector fields. Also, we give new constructions for quasi curvatures of quasi velocity magnetic flows by considering Heisenberg ferromagnetic spin. Finally, the magnetic flux surface is demonstrated in a static and uniform magnetic surface by using the analytical and numerical results. 


\section{Background on the quasi frame}

Let $\Psi=\Psi(s)$ be an arclength parametrized particle in the 3D space $(\mathcal{R}, \cdot)$, i.e. $\left(\Psi_{s} \cdot \Psi_{s}\right)=1$. The arclength parametrized particle is also called a unit speed particle. A unit speed particle $\Psi$ is called to be a Serret particle if $\left\|\Psi_{s s}\right\| \neq 0$. This particle introduces an orthonormal field $(\mathbf{t}, \mathbf{n}, \mathbf{b})$, which satisfies the following formulae

$$
\left[\begin{array}{c}
\nabla_{s} \mathbf{t} \\
\nabla_{s} \mathbf{n} \\
\nabla_{s} \mathbf{b}
\end{array}\right]=\left[\begin{array}{ccc}
0 & \kappa & 0 \\
-\kappa & 0 & \tau \\
0 & -\tau & 0
\end{array}\right]\left[\begin{array}{l}
\mathbf{t} \\
\mathbf{n} \\
\mathbf{b}
\end{array}\right] .
$$

We define a quasi frame $\left(\mathbf{t}^{\mathbf{q}}, \mathbf{n}^{\mathbf{q}}, \mathbf{b}^{\mathbf{q}}\right)$ by only parallel transporting to the tangent vector of the frame along with particle. The quasi directional frame of a regular particle is given by

$$
\mathbf{t}^{\mathbf{q}}=\mathbf{t}, \quad \mathbf{n}^{\mathbf{q}}=\frac{\mathbf{t} \times \pi}{\|\mathbf{t} \times \pi\|}, \quad \mathbf{b}^{\mathbf{q}}=\mathbf{t} \times \mathbf{n}^{\mathbf{q}},
$$

where $\pi$ is a projection vector and can be selected as the following

$$
\pi=(1,0,0) .
$$

If the angle between the quasi normal vecctor $\mathbf{n}^{\mathrm{q}}$ and the normal vector $\mathbf{n}$ is choosen as $\psi$, then the following relation is obtained between the quasi and SF frame $[24,25]$ :

$$
\begin{aligned}
\mathbf{t}_{\mathbf{q}} & =\mathbf{t}, \quad \mathbf{n}^{\mathbf{q}}=\cos \psi \mathbf{n}+\sin \psi \mathbf{b}, \\
\mathbf{b}^{\mathbf{q}} & =-\sin \psi \mathbf{n}+\cos \psi \mathbf{b},
\end{aligned}
$$

Therefore, the quasi frame equations are expressed as

$$
\begin{aligned}
& \nabla_{s} \mathbf{t}_{\mathbf{q}}=\varkappa_{1} \mathbf{n}_{\mathbf{q}}+\varkappa_{2} \mathbf{b}_{\mathbf{q}}, \\
& \nabla_{s} \mathbf{n}_{\mathbf{q}}=-\varkappa_{1} \mathbf{t}_{\mathbf{q}}+\varkappa_{3} \mathbf{b}_{\mathbf{q}}, \\
& \nabla_{s} \mathbf{b}_{\mathbf{q}}=-\varkappa_{2} \mathbf{t}_{\mathbf{q}}-\varkappa_{3} \mathbf{n}_{\mathbf{q}},
\end{aligned}
$$

where the quasi curvatures are

$$
\varkappa_{1}=\kappa \cos \psi, \quad \varkappa_{2}=-\kappa \sin \psi, \quad \varkappa_{3}=\psi^{\prime}+\tau .
$$

\section{Flows of velocity magnetic particles}

In this section, magnetic surfaces with the time evolution of quasi-velocity magnetic partices in 3D space are described. The time evolution is assumed to be one-dimensional and embedded in the 3D space. Thus, the fundamental geometric construction of the flows as surfaces can naturally be induced by the moving quasi orthonormal frame field.

a Let $\alpha$ be an arclength parametrized particle and $\mathcal{B}$ be a magnetic field in the ordinary space. We call the particle $\alpha$ as a quasi-velocity magnetic particle if the quasi tangent field of the particle meets the subsequent Lorentz force equation:

$$
\nabla_{\mathbf{t}_{\mathbf{q}}} \mathbf{t}_{\mathbf{q}}=\phi\left(\mathbf{t}_{\mathbf{q}}\right)=\mathcal{B} \times \mathbf{t}_{\mathbf{q}}
$$

Lorentz force $\phi$ of the magnetic field $\mathcal{B}$ with the quasivelocity magnetic particle is given in the quasi frame by the subsequent equations.

$$
\begin{aligned}
& \phi\left(\mathbf{t}_{\mathbf{q}}\right)=\varkappa_{1} \mathbf{n}_{\mathbf{q}}+\varkappa_{2} \mathbf{b}_{\mathbf{q}}, \\
& \phi\left(\mathbf{n}_{\mathbf{q}}\right)=-\varkappa_{1} \mathbf{t}_{\mathbf{q}}+\varpi \mathbf{b}_{\mathbf{q}}, \\
& \phi\left(\mathbf{b}_{\mathbf{q}}\right)=-\varkappa_{2} \mathbf{t}_{\mathbf{q}}-\varpi \mathbf{n}_{\mathbf{q}},
\end{aligned}
$$

where $\varpi=\phi\left(\mathbf{n}_{\mathbf{q}}\right) \cdot \mathbf{b}_{\mathbf{q}}$ is a sufficiently smooth function and $\varkappa_{1}, \varkappa_{2}$ are quasi-curvatures. Also, magnetic field $\mathcal{B}$ is given by

$$
\mathcal{B}=\varpi \mathbf{t}_{\mathbf{q}}-\varkappa_{2} \mathbf{n}_{\mathbf{q}}+\varkappa_{1} \mathbf{b}_{\mathbf{q}} .
$$

Let $\alpha(s, \omega, t)$ be the motion of regular particles in space. The flow of $\alpha$ can easily be displayed seeing as

$$
\frac{\partial \alpha}{\partial t}=\beta_{1} \mathbf{t}_{\mathbf{q}}+\beta_{2} \mathbf{n}_{\mathbf{q}}+\beta_{3} \mathbf{b}_{\mathbf{q}},
$$

where $\beta_{1}, \beta_{2}, \beta_{3}$ are tangential, normal and binormal potentials of particle.

\$ Time derivatives of the quasi frame are given by

$$
\begin{aligned}
\nabla_{t} \mathbf{t}_{\mathbf{q}} & =\left(\frac{\partial \beta_{2}}{\partial s}+\varkappa_{1} \beta_{1}-\varkappa_{3} \beta_{3}\right) \mathbf{n}_{\mathbf{q}} \\
& +\left(\frac{\partial \beta_{3}}{\partial s}+\varkappa_{2} \beta_{1}+\varkappa_{3} \beta_{2}\right) \mathbf{b}_{\mathbf{q}}, \\
\nabla_{t} \mathbf{n}_{\mathbf{q}}= & -\left(\frac{\partial \beta_{2}}{\partial s}+\varkappa_{1} \beta_{1}-\varkappa_{3} \beta_{3}\right) \mathbf{t}_{\mathbf{q}}+\chi \mathbf{b}_{\mathbf{q}}, \\
\nabla_{t} \mathbf{b}_{\mathbf{q}}= & -\left(\frac{\partial \beta_{3}}{\partial s}+\varkappa_{2} \beta_{1}+\varkappa_{3} \beta_{2}\right) \mathbf{t}_{\mathbf{q}}-\chi \mathbf{n}_{\mathbf{q}},
\end{aligned}
$$

where $\chi=\nabla_{t} \mathbf{n}_{\mathbf{q}} \cdot \mathbf{b}_{\mathbf{q}}$.

\$ Differentation of Lorentz forces are obtained by

$$
\begin{aligned}
& \nabla_{s} \phi\left(\mathbf{t}_{\mathbf{q}}\right)=-\left(\varkappa_{1}^{2}+\varkappa_{2}^{2}\right) \mathbf{t}_{\mathbf{q}}+\left(\varkappa_{1}^{\prime}-\varkappa_{2} \varkappa_{3}\right) \mathbf{n}_{\mathbf{q}}+\left(\varkappa_{2}^{\prime}+\varkappa_{1} \varkappa_{3}\right) \mathbf{b}_{\mathbf{q}}, \\
& \nabla_{s} \phi\left(\mathbf{n}_{\mathbf{q}}\right)=-\left(\varkappa_{1}^{\prime}+\varkappa_{2} \varpi\right) \mathbf{t}_{\mathbf{q}}-\left(\varkappa_{1}^{2}+\varkappa_{3} \varpi\right) \mathbf{n}_{\mathbf{q}}+\left(\varpi^{\prime}-\varkappa_{1} \varkappa_{2}\right) \mathbf{b}_{\mathbf{q}}, \\
& \nabla_{s} \phi\left(\mathbf{b}_{\mathbf{q}}\right)=\left(\varpi \varkappa_{1}-\varkappa_{2}^{\prime}\right) \mathbf{t}_{\mathbf{q}}-\left(\varpi^{\prime}+\varkappa_{2} \varkappa_{1}\right) \mathbf{n}_{\mathbf{q}}-\left(\varkappa_{2}^{2}+\varpi \varkappa_{3}\right) \mathbf{b}_{\mathbf{q}} .
\end{aligned}
$$

by

Flows of Lorentz forces of the quasi frame are given

$$
\begin{aligned}
\nabla_{t} \phi\left(\mathbf{t}_{\mathbf{q}}\right) & =-\left(\varkappa_{1}\left[\frac{\partial \beta_{2}}{\partial s}+\varkappa_{1} \beta_{1}-\varkappa_{3} \beta_{3}\right]\right. \\
& \left.+\varkappa_{2}\left[\frac{\partial \beta_{3}}{\partial s}+\varkappa_{2} \beta_{1}+\varkappa_{3} \beta_{2}\right]\right) \mathbf{t}_{\mathbf{q}} \\
& +\left(\frac{\partial \varkappa_{1}}{\partial t}-\varkappa_{2} \chi\right) \mathbf{n}_{\mathbf{q}}+\left(\frac{\partial \varkappa_{2}}{\partial t}+\varkappa_{1} \chi\right) \mathbf{b}_{\mathbf{q}}, \\
\nabla_{t} \phi\left(\mathbf{n}_{\mathbf{q}}\right) & =-\left(\frac{\partial \varkappa_{1}}{\partial t}+\varpi\left[\frac{\partial \beta_{3}}{\partial s}+\varkappa_{2} \beta_{1}+\varkappa_{3} \beta_{2}\right]\right) \mathbf{t}_{\mathbf{q}} \\
& -\left(\varkappa_{1}\left[\frac{\partial \beta_{2}}{\partial s}+\varkappa_{1} \beta_{1}-\varkappa_{3} \beta_{3}\right]+\varpi \chi\right) \mathbf{n}_{\mathbf{q}} \\
& +\left(\frac{\partial \varpi}{\partial t}-\varkappa_{1}\left[\frac{\partial \beta_{3}}{\partial s}+\varkappa_{2} \beta_{1}+\varkappa_{3} \beta_{2}\right]\right) \mathbf{b}_{\mathbf{q}},
\end{aligned}
$$




$$
\begin{aligned}
\nabla_{t} \phi\left(\mathbf{b}_{\mathbf{q}}\right) & =\left(\varpi\left[\frac{\partial \beta_{2}}{\partial s}+\varkappa_{1} \beta_{1}-\varkappa_{3} \beta_{3}\right]-\frac{\partial \varkappa_{2}}{\partial t}\right) \mathbf{t}_{\mathbf{q}} \\
& -\left(\frac{\partial \varpi}{\partial t}+\varkappa_{2}\left[\frac{\partial \beta_{2}}{\partial s}+\varkappa_{1} \beta_{1}-\varkappa_{3} \beta_{3}\right]\right) \mathbf{n}_{\mathbf{q}} \\
& -\left(\varkappa_{2}\left[\frac{\partial \beta_{3}}{\partial s}+\varkappa_{2} \beta_{1}+\varkappa_{3} \beta_{2}\right]+\varpi \chi\right) \mathbf{b}_{\mathbf{q}} .
\end{aligned}
$$

\section{Quasi magnetic Lorentz flux surfaces}

The magnetic flux equation theory or the theory of flux systems has had an enormous impact on applied physical and mathematical studies. This framework combines some subtle techniques in nonlinear flux optics, field designs and sigma models, fluid dynamics, relativity, and electromagnetic wave theory. In this section, we obtain the condition of quasi magnetic $\phi\left(\mathbf{t}_{\mathbf{q}}\right), \phi\left(\mathbf{n}_{\mathbf{q}}\right), \phi\left(\mathbf{b}_{\mathbf{q}}\right)$ flux by using the Heisenberg ferromagnetic model. These models correspond to the action of point charged particles along with the singular magnetic lines on the spheres or three-dimensional surfaces. Here, we also assume for the rest of the paper that the flow of the magnetic flux between two distinct points on a given surface causes the exact same angular momentum for the charged particle. As opposed to the traditional spectral analysis approach, which is used to built magnetic flux surfaces at small scales we rather choose to focus on the geometric methods in order to avoid the flaws of the aforementioned method, i.e., the nondeterministic polynomial of exponential complexity.

Magnetic $\phi\left(\mathbf{t}_{\mathbf{q}}\right), \phi\left(\mathbf{n}_{\mathbf{q}}\right), \phi\left(\mathbf{b}_{\mathbf{q}}\right)$ flux surfaces afford typical characterizations of connectivity, dynamics, and the spatial structure of magnetic fields, magnetic field connectivity in the interstellar medium and in the interplanetary and dynamo problems, in laboratory plasmas, and magnetic reconnection. Here, the different kinds of magnetic flux surfaces are computed through the modifications of the evolution equations, which define the motion of magnetically driven particles and corresponding gradient flows. We mainly consider the orthonormal curvilinear coordinates and derive the solution families of the equations of the Lorentz force associated with magnetic flux surfaces of the distinct kinds. As will be seen in the next section all three kinds of flux surfaces formed by quasi-tangential, quasi-normal, quasibinormal vectors have different configurations and particular behaviors.

\section{Case 1. Magnetic $\phi\left(\mathbf{t}_{\mathbf{q}}\right)$ flux}

\$ The magnetic $\phi\left(\mathbf{t}_{\mathbf{q}}\right)$ flux ${ }^{m} \mathcal{F}_{\phi\left(\mathbf{t}_{\mathbf{q}}\right)}$ is given by

$$
\begin{aligned}
{ }^{m} \mathcal{F}_{\phi\left(\mathbf{t}_{\mathbf{q}}\right)} & =\int_{\mathcal{F}}\left(\left[\left\{\frac{\partial \varkappa_{1}}{\partial s}-\varkappa_{2} \varkappa_{3}\right\}\left\{\frac{\partial \varkappa_{2}}{\partial t}+\varkappa_{1} \chi\right\}-\left\{\frac{\partial \varkappa_{2}}{\partial s}+\varkappa_{1} \varkappa_{3}\right\}\left\{\frac{\partial \varkappa_{1}}{\partial t}-\varkappa_{2} \chi\right\} \varpi\right.\right. \\
& -\varkappa_{2}\left[\left\{\varkappa_{1}^{2}+\varkappa_{2}^{2}\right\}\left\{\frac{\partial \varkappa_{2}}{\partial t}+\varkappa_{1} \chi\right\}-\left\{\frac{\partial \varkappa_{2}}{\partial s}+\varkappa_{1} \varkappa_{3}\right\}\left[\varkappa_{1}\left\{\frac{\partial \beta_{2}}{\partial s}+\varkappa_{1} \beta_{1}-\varkappa_{3} \beta_{3}\right\}\right.\right. \\
& \left.\left.+\varkappa_{2}\left\{\frac{\partial \beta_{3}}{\partial s}+\varkappa_{2} \beta_{1}+\varkappa_{3} \beta_{2}\right\}\right]\right)+\left[\{ \frac { \partial \varkappa _ { 1 } } { \partial s } - \varkappa _ { 2 } \varkappa _ { 3 } \} \left[\varkappa_{1}\left\{\frac{\partial \beta_{2}}{\partial s}+\varkappa_{1} \beta_{1}-\varkappa_{3} \beta_{3}\right\}\right.\right. \\
& \left.\left.\left.+\varkappa_{2}\left\{\frac{\partial \beta_{3}}{\partial s}+\varkappa_{2} \beta_{1}+\varkappa_{3} \beta_{2}\right\}\right]-\left\{\varkappa_{1}^{2}+\varkappa_{2}^{2}\right\}\left\{\frac{\partial \varkappa_{1}}{\partial t}-\varkappa_{2} \chi\right\}\right] \varkappa_{1}\right) d \pi .
\end{aligned}
$$

Magnetic $\phi\left(\mathbf{t}_{\mathbf{q}}\right)$ flux ${ }^{m} \mathcal{F}_{\phi\left(\mathbf{t}_{\mathbf{q}}\right)}$ is given by

$$
{ }^{m} \mathcal{F}_{\phi\left(\mathbf{t}_{\mathbf{q}}\right)}=\int_{\mathcal{F}} \mathcal{B} \cdot \nabla_{s} \phi\left(\mathbf{t}_{\mathbf{q}}\right) \times \nabla_{t} \phi\left(\mathbf{t}_{\mathbf{q}}\right) d \pi .
$$

With short calculations, we obtain that

$$
\begin{aligned}
\nabla_{s} \phi\left(\mathbf{t}_{\mathbf{q}}\right) \times \nabla_{t} \phi\left(\mathbf{t}_{\mathbf{q}}\right) & =\left(\left[\frac{\partial \varkappa_{1}}{\partial s}-\varkappa_{2} \varkappa_{3}\right]\left[\frac{\partial \varkappa_{2}}{\partial t}+\varkappa_{1} \chi\right]-\left[\frac{\partial \varkappa_{2}}{\partial s}+\varkappa_{1} \varkappa_{3}\right]\left[\frac{\partial \varkappa_{1}}{\partial t}-\varkappa_{2} \chi\right]\right) \mathbf{t}_{\mathbf{q}} \\
& +\left(\left[\varkappa_{1}^{2}+\varkappa_{2}^{2}\right]\left[\frac{\partial \varkappa_{2}}{\partial t}+\varkappa_{1} \chi\right]-\left[\frac{\partial \varkappa_{2}}{\partial s}+\varkappa_{1} \varkappa_{3}\right]\left[\varkappa_{1}\left\{\frac{\partial \beta_{2}}{\partial s}+\varkappa_{1} \beta_{1}-\varkappa_{3} \beta_{3}\right\}\right.\right. \\
& \left.\left.+\varkappa_{2}\left\{\frac{\partial \beta_{3}}{\partial s}+\varkappa_{2} \beta_{1}+\varkappa_{3} \beta_{2}\right\}\right]\right) \mathbf{n}_{\mathbf{q}}+\left([ \frac { \partial \varkappa _ { 1 } } { \partial s } - \varkappa _ { 2 } \varkappa _ { 3 } ] \left[\varkappa_{1}\left\{\frac{\partial \beta_{2}}{\partial s}+\varkappa_{1} \beta_{1}-\varkappa_{3} \beta_{3}\right\}\right.\right. \\
& \left.\left.+\varkappa_{2}\left\{\frac{\partial \beta_{3}}{\partial s}+\varkappa_{2} \beta_{1}+\varkappa_{3} \beta_{2}\right\}\right]-\left[\varkappa_{1}^{2}+\varkappa_{2}^{2}\right]\left[\frac{\partial \varkappa_{1}}{\partial t}-\varkappa_{2} \chi\right]\right) \mathbf{b}_{\mathbf{q}} .
\end{aligned}
$$


NEW MAGNETIC FLUX FLOWS WITH HEISENBERG FERROMAGNETIC SPIN OF OPTICAL QUASI VELOCITY MAGNETIC ...

381

Magnetic flux density of $\phi\left(\mathbf{t}_{\mathbf{q}}\right)$ is given by

$$
\begin{aligned}
{ }^{m} \mathcal{L}_{\phi\left(\mathbf{t}_{\mathbf{q}}\right)} & =\left(\left[\frac{\partial \varkappa_{1}}{\partial s}-\varkappa_{2} \varkappa_{3}\right]\left[\frac{\partial \varkappa_{2}}{\partial t}+\varkappa_{1} \chi\right]-\left[\frac{\partial \varkappa_{2}}{\partial s}+\varkappa_{1} \varkappa_{3}\right]\left[\frac{\partial \varkappa_{1}}{\partial t}-\varkappa_{2} \chi\right]\right) \varpi \\
& -\varkappa_{2}\left(\left[\varkappa_{1}^{2}+\varkappa_{2}^{2}\right]\left[\frac{\partial \varkappa_{2}}{\partial t}+\varkappa_{1} \chi\right]-\left[\frac{\partial \varkappa_{2}}{\partial s}+\varkappa_{1} \varkappa_{3}\right]\left[\varkappa_{1}\left\{\frac{\partial \beta_{2}}{\partial s}+\varkappa_{1} \beta_{1}-\varkappa_{3} \beta_{3}\right\}\right.\right. \\
& \left.\left.+\varkappa_{2}\left\{\frac{\partial \beta_{3}}{\partial s}+\varkappa_{2} \beta_{1}+\varkappa_{3} \beta_{2}\right\}\right]\right)+\left([ \frac { \partial \varkappa _ { 1 } } { \partial s } - \varkappa _ { 2 } \varkappa _ { 3 } ] \left[\varkappa_{1}\left\{\frac{\partial \beta_{2}}{\partial s}+\varkappa_{1} \beta_{1}-\varkappa_{3} \beta_{3}\right\}\right.\right. \\
& \left.\left.+\varkappa_{2}\left\{\frac{\partial \beta_{3}}{\partial s}+\varkappa_{2} \beta_{1}+\varkappa_{3} \beta_{2}\right\}\right]-\left[\varkappa_{1}^{2}+\varkappa_{2}^{2}\right]\left[\frac{\partial \varkappa_{1}}{\partial t}-\varkappa_{2} \chi\right]\right) \varkappa_{1} .
\end{aligned}
$$

Moreover, $\phi\left(\mathbf{t}_{\mathbf{q}}\right)$ flux is obtained in the following way

$$
\begin{aligned}
{ }^{m} \mathcal{F}_{\phi\left(\mathbf{t}_{\mathbf{q}}\right)} & =\int_{\mathcal{F}}\left(\left[\left\{\frac{\partial \varkappa_{1}}{\partial s}-\varkappa_{2} \varkappa_{3}\right\}\left\{\frac{\partial \varkappa_{2}}{\partial t}+\varkappa_{1} \chi\right\}-\left\{\frac{\partial \varkappa_{2}}{\partial s}+\varkappa_{1} \varkappa_{3}\right\}\left\{\frac{\partial \varkappa_{1}}{\partial t}-\varkappa_{2} \chi\right\}\right]\right. \\
& -\varkappa_{2}\left[\left\{\varkappa_{1}^{2}+\varkappa_{2}^{2}\right\}\left\{\frac{\partial \varkappa_{2}}{\partial t}+\varkappa_{1} \chi\right\}-\left\{\frac{\partial \varkappa_{2}}{\partial s}+\varkappa_{1} \varkappa_{3}\right\}\left\{\varkappa_{1}\left(\frac{\partial \beta_{2}}{\partial s}+\varkappa_{1} \beta_{1}-\varkappa_{3} \beta_{3}\right)\right.\right. \\
& \left.\left.+\varkappa_{2}\left(\frac{\partial \beta_{3}}{\partial s}+\varkappa_{2} \beta_{1}+\varkappa_{3} \beta_{2}\right)\right\}\right]+\left[\{ \frac { \partial \varkappa _ { 1 } } { \partial s } - \varkappa _ { 2 } \varkappa _ { 3 } \} \left\{\varkappa_{1}\left(\frac{\partial \beta_{2}}{\partial s}+\varkappa_{1} \beta_{1}-\varkappa_{3} \beta_{3}\right)\right.\right. \\
& \left.\left.\left.+\varkappa_{2}\left(\frac{\partial \beta_{3}}{\partial s}+\varkappa_{2} \beta_{1}+\varkappa_{3} \beta_{2}\right)\right\}-\left\{\varkappa_{1}^{2}+\varkappa_{2}^{2}\right\}\left\{\frac{\partial \varkappa_{1}}{\partial t}-\varkappa_{2} \chi\right\}\right] \varkappa_{1}\right) d \pi .
\end{aligned}
$$

From the ferromagnetic model, the flux density is given by

$$
{ }^{m} \mathcal{L}_{\phi\left(\mathbf{t}_{\mathbf{q}}\right)}^{\mathfrak{f e r r o}}=\mathcal{B} \cdot \nabla_{s} \phi\left(\mathbf{t}_{\mathbf{q}}\right) \times \phi\left(\mathbf{t}_{\mathbf{q}}\right) \times \nabla_{s}^{2} \phi\left(\mathbf{t}_{\mathbf{q}}\right)
$$

Similarly, we can obtain that

$$
\begin{aligned}
\nabla_{s} \phi\left(\mathbf{t}_{\mathbf{q}}\right) \times \phi\left(\mathbf{t}_{\mathbf{q}}\right) \times \nabla_{s}^{2} \phi\left(\mathbf{t}_{\mathbf{q}}\right)=\left([ \varkappa _ { 1 } ^ { \prime } - \varkappa _ { 2 } \varkappa _ { 3 } ] \varkappa _ { 1 } \left[\frac{\partial}{\partial s}\left\{\varkappa_{1}^{2}+\varkappa_{2}^{2}\right\}+\varkappa_{1}\left\{\frac{\partial \varkappa_{1}}{\partial s}-\varkappa_{2} \varkappa_{3}\right\}\right.\right. \\
\left.\left.+\varkappa_{2}\left\{\frac{\partial \varkappa_{2}}{\partial s}+\varkappa_{1} \varkappa_{3}\right\}\right]+\left[\varkappa_{2}^{\prime}+\varkappa_{1} \varkappa_{3}\right] \varkappa_{2}\left[\frac{\partial}{\partial s}\left\{\varkappa_{1}^{2}+\varkappa_{2}^{2}\right\}+\varkappa_{1}\left\{\frac{\partial \varkappa_{1}}{\partial s}-\varkappa_{2} \varkappa_{3}\right\}+\varkappa_{2}\left\{\frac{\partial \varkappa_{2}}{\partial s}+\varkappa_{1} \varkappa_{3}\right\}\right]\right) \mathbf{t}_{\mathbf{q}} \\
+\left(\varkappa_{1}\left[\frac{\partial}{\partial s}\left\{\varkappa_{1}^{2}+\varkappa_{2}^{2}\right\}+\varkappa_{1}\left\{\frac{\partial \varkappa_{1}}{\partial s}-\varkappa_{2} \varkappa_{3}\right\}+\varkappa_{2}\left\{\frac{\partial \varkappa_{2}}{\partial s}+\varkappa_{1} \varkappa_{3}\right\}\right]\left[\varkappa_{1}^{2}+\varkappa_{2}^{2}\right]+\left[\varkappa_{2}^{\prime}+\varkappa_{1} \varkappa_{3}\right]\left[\varkappa _ { 1 } \left\{\frac{\partial}{\partial s}\left[\frac{\partial \varkappa_{2}}{\partial s}+\varkappa_{1} \varkappa_{3}\right]\right.\right.\right. \\
\left.\left.-\left[\varkappa_{1}^{2}+\varkappa_{2}^{2}\right] \varkappa_{2}+\varkappa_{3}\left\{\frac{\partial \varkappa_{1}}{\partial s}-\varkappa_{2} \varkappa_{3}\right\}\right]-\varkappa_{2}\left[\frac{\partial}{\partial s}\left\{\frac{\partial \varkappa_{1}}{\partial s}-\varkappa_{2} \varkappa_{3}\right\}+\varkappa_{3}\left\{\frac{\partial \varkappa_{2}}{\partial s}+\varkappa_{1} \varkappa_{3}\right\}+\left\{\varkappa_{1}^{2}+\varkappa_{2}^{2}\right\} \varkappa_{1}\right]\right) \mathbf{n}_{\mathbf{q}} \\
+\left(\left[\varkappa_{1}^{2}+\varkappa_{2}^{2}\right] \varkappa_{2}\left[\frac{\partial}{\partial s}\left\{\varkappa_{1}^{2}+\varkappa_{2}^{2}\right\}+\varkappa_{1}\left\{\frac{\partial \varkappa_{1}}{\partial s}-\varkappa_{2} \varkappa_{3}\right\}+\varkappa_{2}\left\{\frac{\partial \varkappa_{2}}{\partial s}+\varkappa_{1} \varkappa_{3}\right\}\right]-\left[\varkappa_{1}^{\prime}-\varkappa_{2} \varkappa_{3}\right]\left[\varkappa _ { 1 } \left\{\frac{\partial}{\partial s}\left[\frac{\partial \varkappa_{2}}{\partial s}+\varkappa_{1} \varkappa_{3}\right]\right.\right.\right. \\
\left.\left.\left.-\left\lceil\varkappa_{1}^{2}+\varkappa_{2}^{2}\right\rceil \varkappa_{2}+\varkappa_{3}\left[\frac{\partial \varkappa_{1}}{\partial s}-\varkappa_{2} \varkappa_{3}\right]\right\}-\varkappa_{2}\left[\frac{\partial}{\partial s}\left\{\frac{\partial \varkappa_{1}}{\partial s}-\varkappa_{2} \varkappa_{3}\right\}+\varkappa_{3}\left\{\frac{\partial \varkappa_{2}}{\partial s}+\varkappa_{1} \varkappa_{3}\right\}+\left\{\varkappa_{1}^{2}+\varkappa_{2}^{2}\right\} \varkappa_{1}\right\}\right]\right) \mathbf{b}_{\mathbf{q}} .
\end{aligned}
$$

Rev. Mex. Fís. 67 (3) 378-392 
382

T. KÖ RPINAR, R. CEM DEMIRKOL, Z. KÖ RPINAR, AND V. ASL

Similarly, ferromagnetic magnetic $\phi\left(\mathbf{t}_{\mathbf{q}}\right)$ flux is given by

$$
\begin{aligned}
m_{\mathcal{F}_{\phi\left(\mathbf{t}_{\mathbf{q}}\right)}^{\mathfrak{f e r r}}} & =\int_{\mathcal{F}}\left(\varpi \left[\left\{\varkappa_{1}^{\prime}-\varkappa_{2} \varkappa_{3}\right\} \varkappa_{1}\left\{\frac{\partial}{\partial s}\left(\varkappa_{1}^{2}+\varkappa_{2}^{2}\right)+\varkappa_{1}\left(\frac{\partial \varkappa_{1}}{\partial s}-\varkappa_{2} \varkappa_{3}\right)+\varkappa_{2}\left(\frac{\partial \varkappa_{2}}{\partial s}+\varkappa_{1} \varkappa_{3}\right)\right\}\right.\right. \\
& \left.+\left\{\varkappa_{2}^{\prime}+\varkappa_{1} \varkappa_{3}\right\} \varkappa_{2}\left\{\frac{\partial}{\partial s}\left(\varkappa_{1}^{2}+\varkappa_{2}^{2}\right)+\varkappa_{1}\left(\frac{\partial \varkappa_{1}}{\partial s}-\varkappa_{2} \varkappa_{3}\right)+\varkappa_{2}\left(\frac{\partial \varkappa_{2}}{\partial s}+\varkappa_{1} \varkappa_{3}\right)\right\}\right] \\
& -\varkappa_{2}\left[\varkappa_{1}\left\{\frac{\partial}{\partial s}\left(\varkappa_{1}^{2}+\varkappa_{2}^{2}\right)+\varkappa_{1}\left(\frac{\partial \varkappa_{1}}{\partial s}-\varkappa_{2} \varkappa_{3}\right)+\varkappa_{2}\left(\frac{\partial \varkappa_{2}}{\partial s}+\varkappa_{1} \varkappa_{3}\right)\right\}\left\{\varkappa_{1}^{2}+\varkappa_{2}^{2}\right\}+\left\{\varkappa_{2}^{\prime}+\varkappa_{1} \varkappa_{3}\right\}\right. \\
& \times\left\{\varkappa _ { 1 } \left(\frac{\partial}{\partial s}\left[-\left[\frac{\partial \varkappa_{2}}{\partial s}+\varkappa_{1} \varkappa_{3}\right]-\left[\varkappa_{1}^{2}+\varkappa_{2}^{2}\right] \varkappa_{2}+\varkappa_{3}-\left[\frac{\partial \varkappa_{1}}{\partial s}-\varkappa_{2} \varkappa_{3}\right]\right)-\varkappa_{2}\left(\frac{\partial}{\partial s}\left[\frac{\partial \varkappa_{1}}{\partial s}-\varkappa_{2} \varkappa_{3}\right]\right.\right.\right. \\
& \left.\left.\left.+\varkappa_{3}\left[\frac{\partial \varkappa_{2}}{\partial s}+\varkappa_{1} \varkappa_{3}\right]+\left[\varkappa_{1}^{2}+\varkappa_{2}^{2}\right] \varkappa_{1}\right)\right\}\right]+\varkappa_{1}\left[\{ \varkappa _ { 1 } ^ { 2 } + \varkappa _ { 2 } ^ { 2 } \} \varkappa _ { 2 } \left\{\frac{\partial}{\partial s}\left(\varkappa_{1}^{2}+\varkappa_{2}^{2}\right)+\varkappa_{1}\left(\frac{\partial \varkappa_{1}}{\partial s}-\varkappa_{2} \varkappa_{3}\right)\right.\right. \\
& \left.+\varkappa_{2}\left(\frac{\partial \varkappa_{2}}{\partial s}+\varkappa_{1} \varkappa_{3}\right)\right\}-\left\{\varkappa_{1}^{\prime}-\varkappa_{2} \varkappa_{3}\right\}\left\{\varkappa_{1}\left(\frac{\partial}{\partial s}\left[\frac{\partial \varkappa_{2}}{\partial s} \varkappa_{1} \varkappa_{3}\right]-\left[\varkappa_{1}^{2}+\varkappa_{2}^{2}\right] \varkappa_{2}+\varkappa_{3}-\left[\frac{\partial \varkappa_{1}}{\partial s}-\varkappa_{2} \varkappa_{3}\right]\right)\right. \\
& \left.\left.\left.-\varkappa_{2}\left(\frac{\partial}{\partial s}\left[\frac{\partial \varkappa_{1}}{\partial s}-\varkappa_{2} \varkappa_{3}\right]+\varkappa_{3}-\left[\frac{\partial \varkappa_{2}}{\partial s}+\varkappa_{1} \varkappa_{3}\right]+\left[\varkappa_{1}^{2}+\varkappa_{2}^{2}\right] \varkappa_{1}\right)\right\}\right]\right) d \pi .
\end{aligned}
$$

The magnetic $\phi\left(\mathbf{t}_{\mathbf{q}}\right)$ flux surface condition is given by

$$
\begin{aligned}
& \left(\left[\frac{\partial \varkappa_{1}}{\partial s}-\varkappa_{2} \varkappa_{3}\right]\left[\frac{\partial \varkappa_{2}}{\partial t}+\varkappa_{1} \chi\right]-\left[\frac{\partial \varkappa_{2}}{\partial s}+\varkappa_{1} \varkappa_{3}\right]\left[\frac{\partial \varkappa_{1}}{\partial t}-\varkappa_{2} \chi\right]\right) \varpi \\
& -\varkappa_{2}\left(\left(\varkappa_{1}^{2}+\varkappa_{2}^{2}\right)\left[\frac{\partial \varkappa_{2}}{\partial t}+\varkappa_{1} \chi\right]-\left[\frac{\partial \varkappa_{2}}{\partial s}+\varkappa_{1} \varkappa_{3}\right]\left[\varkappa_{1}\left\{\frac{\partial \beta_{2}}{\partial s}+\varkappa_{1} \beta_{1}-\varkappa_{3} \beta_{3}\right\}\right.\right. \\
& \left.\left.+\varkappa_{2}\left\{\frac{\partial \beta_{3}}{\partial s}+\varkappa_{2} \beta_{1}+\varkappa_{3} \beta_{2}\right\}\right]\right)+\left([ \frac { \partial \varkappa _ { 1 } } { \partial s } - \varkappa _ { 2 } \varkappa _ { 3 } ] \left[\varkappa_{1}\left\{\frac{\partial \beta_{2}}{\partial s}+\varkappa_{1} \beta_{1}-\varkappa_{3} \beta_{3}\right\}\right.\right. \\
& \left.\left.+\varkappa_{2}\left\{\frac{\partial \beta_{3}}{\partial s}+\varkappa_{2} \beta_{1}+\varkappa_{3} \beta_{2}\right\}\right]-\left(\varkappa_{1}^{2}+\varkappa_{2}^{2}\right)\left[\frac{\partial \varkappa_{1}}{\partial t}-\varkappa_{2} \chi\right]\right) \varkappa_{1}=0 .
\end{aligned}
$$

The magnetic $\phi\left(\mathbf{t}_{\mathbf{q}}\right)$ flux surface is given by the ferromagnetic condition

$$
\begin{aligned}
\varpi\left(\left[\varkappa_{1}^{\prime}-\varkappa_{2} \varkappa_{3}\right]\right. & \varkappa_{1}\left[\frac{\partial}{\partial s}\left\{\varkappa_{1}^{2}+\varkappa_{2}^{2}\right\}+\varkappa_{1}\left\{\frac{\partial \varkappa_{1}}{\partial s}-\varkappa_{2} \varkappa_{3}\right\}+\varkappa_{2}\left\{\frac{\partial \varkappa_{2}}{\partial s}+\varkappa_{1} \varkappa_{3}\right\}\right]+\left[\varkappa_{2}^{\prime}+\varkappa_{1} \varkappa_{3}\right] \varkappa_{2} \\
\times & {\left.\left[\frac{\partial}{\partial s}\left\{\varkappa_{1}^{2}+\varkappa_{2}^{2}\right\}+\varkappa_{1}\left\{\frac{\partial \varkappa_{1}}{\partial s}-\varkappa_{2} \varkappa_{3}\right\}+\varkappa_{2}\left\{\frac{\partial \varkappa_{2}}{\partial s}+\varkappa_{1} \varkappa_{3}\right\}\right]\right)-\varkappa_{2}\left(\varkappa _ { 1 } \left[\frac{\partial}{\partial s}\left\{\varkappa_{1}^{2}+\varkappa_{2}^{2}\right\}\right.\right.} \\
& \left.+\varkappa_{1}\left\{\frac{\partial \varkappa_{1}}{\partial s}-\varkappa_{2} \varkappa_{3}\right\}+\varkappa_{2}\left\{\frac{\partial \varkappa_{2}}{\partial s}+\varkappa_{1} \varkappa_{3}\right\}\right]\left[\varkappa_{1}^{2}+\varkappa_{2}^{2}\right]+\left[\varkappa_{2}^{\prime}+\varkappa_{1} \varkappa_{3}\right]\left[\varkappa _ { 1 } \left\{\frac{\partial}{\partial s}\left(\frac{\partial \varkappa_{2}}{\partial s}+\varkappa_{1} \varkappa_{3}\right)\right.\right. \\
& \left.-\left(\varkappa_{1}^{2}+\varkappa_{2}^{2}\right) \varkappa_{2}+\varkappa_{3}\left(\frac{\partial \varkappa_{1}}{\partial s}-\varkappa_{2} \varkappa_{3}\right)\right\}-\varkappa_{2}\left\{\frac{\partial}{\partial s}\left(\frac{\partial \varkappa_{1}}{\partial s}-\varkappa_{2} \varkappa_{3}\right)+\varkappa_{3}\left(\frac{\partial \varkappa_{2}}{\partial s}+\varkappa_{1} \varkappa_{3}\right)\right. \\
& \left.\left.\left.+\left(\varkappa_{1}^{2}+\varkappa_{2}^{2}\right) \varkappa_{1}\right\}\right]\right)+\varkappa_{1}\left(\left[\varkappa_{1}^{2}+\varkappa_{2}^{2}\right] \varkappa_{2}\left[\frac{\partial}{\partial s}\left\{\varkappa_{1}^{2}+\varkappa_{2}^{2}\right\}+\varkappa_{1}\left\{\frac{\partial \varkappa_{1}}{\partial s}-\varkappa_{2} \varkappa_{3}\right\}+\varkappa_{2}\left\{\frac{\partial \varkappa_{2}}{\partial s}+\varkappa_{1} \varkappa_{3}\right\}\right]\right. \\
& -\left[\varkappa_{1}^{\prime}-\varkappa_{2} \varkappa_{3}\right]\left[\varkappa_{1}\left\{\frac{\partial}{\partial s}\left(\frac{\partial \varkappa_{2}}{\partial s}+\varkappa_{1} \varkappa_{3}\right)-\left(\varkappa_{1}^{2}+\varkappa_{2}^{2}\right) \varkappa_{2}+\varkappa_{3}\left(\frac{\partial \varkappa_{1}}{\partial s}-\varkappa_{2} \varkappa_{3}\right)\right\}\right. \\
& \left.\left.-\varkappa_{2}\left\{\frac{\partial}{\partial s}\left(\frac{\partial \varkappa_{1}}{\partial s}-\varkappa_{2} \varkappa_{3}\right)+\varkappa_{3}\left(\frac{\partial \varkappa_{2}}{\partial s}+\varkappa_{1} \varkappa_{3}\right)+\left(\varkappa_{1}^{2}+\varkappa_{2}^{2}\right) \varkappa_{1}\right\}\right]\right)=0 .
\end{aligned}
$$

Rev. Mex. Fís. 67 (3) 378-392 


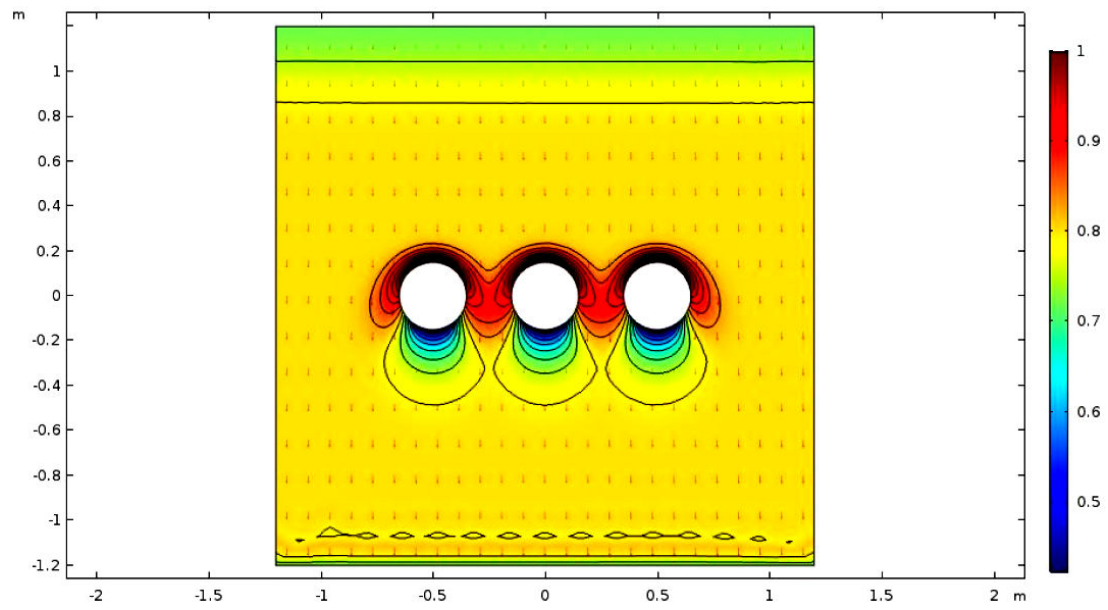

FIGURE 1. The magnetic flux density with Lorentz force $\phi\left(\mathbf{t}_{\mathbf{q}}\right)$.

The magnetic flux density and flow lines in the axis is represented by quadrupole magnets under the action of the Lorentz force $\phi\left(\mathbf{t}_{\mathbf{q}}\right)$. The magnetic flux density on time of flight is fed to the particle tracing algorithm by a logical expression. Here, the intricacy of the magnetic flux surface is demonstrated in a static and uniform magnetic surface by using the analytical and numerical results. To obtain the visualization of the evolved systems of the magnetic $\phi\left(\mathbf{t}_{\mathbf{q}}\right)$ flux we use the basic numerical algorithms to solve the above equations at Matlab and Comsol software. This technique presents a new approach to formulating the relationship between local frames of reference and flux coordinates in Fig. 1.

Case 2. Magnetic $\phi\left(\mathbf{n}_{\mathbf{q}}\right)$ flux

The magnetic $\phi\left(\mathbf{n}_{\mathbf{q}}\right)$ flux ${ }^{m} \mathcal{F}_{\phi\left(\mathbf{n}_{\mathbf{q}}\right)}$ is given by

$$
\begin{aligned}
{ }^{m} \mathcal{F}_{\phi\left(\mathbf{n}_{\mathbf{q}}\right)} & =\int_{\mathcal{F}}\left(\left[\left\{\frac{\partial \varpi}{\partial s}-\varkappa_{1} \varkappa_{2}\right\}\left\{\varkappa_{1}\left(\frac{\partial \beta_{2}}{\partial s}+\varkappa_{1} \beta_{1}-\varkappa_{3} \beta_{3}\right)+\varpi \chi\right\}-\left\{\varkappa_{1}^{2}+\varkappa_{3} \varpi\right\}\right.\right. \\
& \left.\left\{\frac{\partial \varpi}{\partial t}-\varkappa_{1}\left(\frac{\partial \beta_{3}}{\partial s}+\varkappa_{2} \beta_{1}+\varkappa_{3} \beta_{2}\right)\right\}\right] \varpi-\varkappa_{2}\left[\{ \frac { \partial \varkappa _ { 1 } } { \partial s } + \varkappa _ { 2 } \varpi \} \left\{\frac{\partial \varpi}{\partial t}\right.\right. \\
& \left.\left.-\varkappa_{1}\left(\frac{\partial \beta_{3}}{\partial s}+\varkappa_{2} \beta_{1}+\varkappa_{3} \beta_{2}\right)\right\}-\left\{\frac{\partial \varpi}{\partial s}-\varkappa_{1} \varkappa_{2}\right\}\left\{\frac{\partial \varkappa_{1}}{\partial t}+\varpi\left(\frac{\partial \beta_{3}}{\partial s}+\varkappa_{2} \beta_{1}+\varkappa_{3} \beta_{2}\right)\right\}\right] \\
& +\varkappa_{1}\left[\left\{\frac{\partial \varkappa_{1}}{\partial s}+\varkappa_{2} \varpi\right\}\left\{\varkappa_{1}\left(\frac{\partial \beta_{2}}{\partial s}+\varkappa_{1} \beta_{1}-\varkappa_{3} \beta_{3}\right)+\varpi \chi\right\}\right. \\
& \left.\left.-\left\{\varkappa_{1}^{2}+\varkappa_{3} \varpi\right\}\left\{\frac{\partial \varkappa_{1}}{\partial t}+\varpi\left(\frac{\partial \beta_{3}}{\partial s}+\varkappa_{2} \beta_{1}+\varkappa_{3} \beta_{2}\right)\right\}\right]\right) d \pi .
\end{aligned}
$$

Firstly, we compute that

$$
\begin{aligned}
\nabla_{s}^{2} \phi\left(\mathbf{n}_{\mathbf{q}}\right) & =\left(\varkappa_{1}\left[\varkappa_{1}^{2}+\varkappa_{3} \varpi\right]-\frac{\partial}{\partial s}\left[\frac{\partial \varkappa_{1}}{\partial s}+\varkappa_{2} \varpi\right]-\varkappa_{2}\left[\frac{\partial \varpi}{\partial s}-\varkappa_{1} \varkappa_{2}\right]\right) \mathbf{t}_{\mathbf{q}} \\
& -\left(\frac{\partial}{\partial s}\left[\varkappa_{1}^{2}+\varkappa_{3} \varpi\right]+\left[\frac{\partial \varkappa_{1}}{\partial s}+\varkappa_{2} \varpi\right] \varkappa_{1}+\varkappa_{3}\left[\frac{\partial \varpi}{\partial s}-\varkappa_{1} \varkappa_{2}\right]\right) \mathbf{n}_{\mathbf{q}} \\
& +\left(\frac{\partial}{\partial s}\left[\frac{\partial \varpi}{\partial s}-\varkappa_{1} \varkappa_{2}\right]-\left[\frac{\partial \varkappa_{1}}{\partial s}+\varkappa_{2} \varpi\right] \varkappa_{2}-\left[\varkappa_{1}^{2}+\varkappa_{3} \varpi\right] \varkappa_{3}\right) \mathbf{b}_{\mathbf{q}} .
\end{aligned}
$$


Then, it is easy to see that

$$
\begin{aligned}
\phi\left(\mathbf{n}_{\mathbf{q}}\right) \times \nabla_{s}^{2} \phi\left(\mathbf{n}_{\mathbf{q}}\right) & =\varpi\left(\frac{\partial}{\partial s}\left[\varkappa_{1}^{2}+\varkappa_{3} \varpi\right]+\left[\frac{\partial \varkappa_{1}}{\partial s}+\varkappa_{2} \varpi\right] \varkappa_{1}+\varkappa_{3}\left[\frac{\partial \varpi}{\partial s}-\varkappa_{1} \varkappa_{2}\right]\right) \mathbf{t}_{\mathbf{q}} \\
& +\left(\varkappa_{1}\left[\frac{\partial}{\partial s}\left\{\frac{\partial \varpi}{\partial s}-\varkappa_{1} \varkappa_{2}\right\}-\left\{\frac{\partial \varkappa_{1}}{\partial s}+\varkappa_{2} \varpi\right\} \varkappa_{2}-\left\{\varkappa_{1}^{2}+\varkappa_{3} \varpi\right\} \varkappa_{3}\right]\right. \\
& \left.+\varpi\left[\varkappa_{1}\left\{\varkappa_{1}^{2}+\varkappa_{3} \varpi\right\}-\frac{\partial}{\partial s}\left\{\frac{\partial \varkappa_{1}}{\partial s}+\varkappa_{2} \varpi\right\}-\varkappa_{2}\left\{\frac{\partial \varpi}{\partial s}-\varkappa_{1} \varkappa_{2}\right\}\right]\right) \mathbf{n}_{\mathbf{q}} \\
& +\varkappa_{1}\left(\frac{\partial}{\partial s}\left[\varkappa_{1}^{2}+\varkappa_{3} \varpi\right]+\left[\frac{\partial \varkappa_{1}}{\partial s}+\varkappa_{2} \varpi\right] \varkappa_{1}+\varkappa_{3}\left[\frac{\partial \varpi}{\partial s}-\varkappa_{1} \varkappa_{2}\right]\right) \mathbf{b}_{\mathbf{q}} .
\end{aligned}
$$

It is also true that

$$
\begin{aligned}
\nabla_{t} \phi\left(\mathbf{n}_{\mathbf{q}}\right) & =-\left(\frac{\partial \varkappa_{1}}{\partial t}+\varpi\left[\frac{\partial \beta_{3}}{\partial s}+\varkappa_{2} \beta_{1}+\varkappa_{3} \beta_{2}\right]\right) \mathbf{t}_{\mathbf{q}}-\left(\varkappa_{1}\left[\frac{\partial \beta_{2}}{\partial s}+\varkappa_{1} \beta_{1}-\varkappa_{3} \beta_{3}\right]+\varpi \chi\right) \mathbf{n}_{\mathbf{q}} \\
& +\left(\frac{\partial \varpi}{\partial t}-\varkappa_{1}\left[\frac{\partial \beta_{3}}{\partial s}+\varkappa_{2} \beta_{1}+\varkappa_{3} \beta_{2}\right]\right) \mathbf{b}_{\mathbf{q}} .
\end{aligned}
$$

Thus, we can easily obtain that

$$
\begin{aligned}
\nabla_{s} \phi\left(\mathbf{n}_{\mathbf{q}}\right) \times \nabla_{t} \phi\left(\mathbf{n}_{\mathbf{q}}\right) & =\left(\left[\frac{\partial \varpi}{\partial s}-\varkappa_{1} \varkappa_{2}\right]\left[\varkappa_{1}\left\{\frac{\partial \beta_{2}}{\partial s}+\varkappa_{1} \beta_{1}-\varkappa_{3} \beta_{3}\right\}+\varpi \chi\right]-\left(\varkappa_{1}^{2}+\varkappa_{3} \varpi\right)\left[\frac{\partial \varpi}{\partial t}\right.\right. \\
& \left.\left.-\varkappa_{1}\left\{\frac{\partial \beta_{3}}{\partial s}+\varkappa_{2} \beta_{1}+\varkappa_{3} \beta_{2}\right\}\right]\right) \mathbf{t}_{\mathbf{q}}+\left(\left[\frac{\partial \varkappa_{1}}{\partial s}+\varkappa_{2} \varpi\right]\left[\frac{\partial \varpi}{\partial t}-\varkappa_{1}\left\{\frac{\partial \beta_{3}}{\partial s}+\varkappa_{2} \beta_{1}+\varkappa_{3} \beta_{2}\right\}\right]\right. \\
& \left.-\left[\frac{\partial \varpi}{\partial s}-\varkappa_{1} \varkappa_{2}\right] \frac{\partial \varkappa_{1}}{\partial t}+\varpi\left[\frac{\partial \beta_{3}}{\partial s}+\varkappa_{2} \beta_{1}+\varkappa_{3} \beta_{2}\right]\right) \mathbf{n}_{\mathbf{q}}+\left([ \frac { \partial \varkappa _ { 1 } } { \partial s } + \varkappa _ { 2 } \varpi ] \left[\varkappa_{1}\left\{\frac{\partial \beta_{2}}{\partial s}+\varkappa_{1} \beta_{1}-\varkappa_{3} \beta_{3}\right\}\right.\right. \\
& \left.+\varpi \chi]-\left(\varkappa_{1}^{2}+\varkappa_{3} \varpi\right)\left[\frac{\partial \varkappa_{1}}{\partial t}+\varpi\left\{\frac{\partial \beta_{3}}{\partial s}+\varkappa_{2} \beta_{1}+\varkappa_{3} \beta_{2}\right\}\right]\right) \mathbf{b}_{\mathbf{q}} .
\end{aligned}
$$

As a result, we reach the following identities

$$
\begin{aligned}
{ }^{m} \mathcal{L}_{\phi\left(\mathbf{n}_{\mathbf{q}}\right)} & =\left(\left[\frac{\partial \varpi}{\partial s}-\varkappa_{1} \varkappa_{2}\right]\left[\varkappa_{1}\left\{\frac{\partial \beta_{2}}{\partial s}+\varkappa_{1} \beta_{1}-\varkappa_{3} \beta_{3}\right\}+\varpi \chi\right]-\left(\varkappa_{1}^{2}+\varkappa_{3} \varpi\right)\left[\frac{\partial \varpi}{\partial t}-\varkappa_{1}\left\{\frac{\partial \beta_{3}}{\partial s}\right.\right.\right. \\
& \left.\left.\left.+\varkappa_{2} \beta_{1}+\varkappa_{3} \beta_{2}\right\}\right]\right) \varpi-\varkappa_{2}\left(\left[\frac{\partial \varkappa_{1}}{\partial s}+\varkappa_{2} \varpi\right]\left[\frac{\partial \varpi}{\partial t}-\varkappa_{1}\left\{\frac{\partial \beta_{3}}{\partial s}+\varkappa_{2} \beta_{1}+\varkappa_{3} \beta_{2}\right\}\right]\right. \\
& \left.-\left[\frac{\partial \varpi}{\partial s}-\varkappa_{1} \varkappa_{2}\right]\left[\frac{\partial \varkappa_{1}}{\partial t}+\varpi\left\{\frac{\partial \beta_{3}}{\partial s}+\varkappa_{2} \beta_{1}+\varkappa_{3} \beta_{2}\right\}\right]\right)+\varkappa_{1}\left(\left[\frac{\partial \varkappa_{1}}{\partial s}+\varkappa_{2} \varpi\right]\right. \\
& \left.\times\left[\varkappa_{1}\left\{\frac{\partial \beta_{2}}{\partial s}+\varkappa_{1} \beta_{1}-\varkappa_{3} \beta_{3}\right\}+\varpi \chi\right]-\left(\varkappa_{1}^{2}+\varkappa_{3} \varpi\right)\left[\frac{\partial \varkappa_{1}}{\partial t}+\varpi\left\{\frac{\partial \beta_{3}}{\partial s}+\varkappa_{2} \beta_{1}+\varkappa_{3} \beta_{2}\right\}\right]\right) .
\end{aligned}
$$

By ferromagnetic spin for $\phi\left(\mathbf{n}_{\mathbf{q}}\right)$, we obtain

$$
{ }^{m} \mathcal{L}_{\phi\left(\mathbf{n}_{\mathbf{q}}\right)}^{\mathfrak{f e r r o}}=\mathcal{B} \cdot \nabla_{s} \phi\left(\mathbf{n}_{\mathbf{q}}\right) \times \phi\left(\mathbf{n}_{\mathbf{q}}\right) \times \nabla_{s}^{2} \phi\left(\mathbf{n}_{\mathbf{q}}\right)
$$


Therefore, under the assumptions of the Heisenberg ferromagnetic model, it can be computed that

$$
\begin{aligned}
\nabla_{s} \phi\left(\mathbf{n}_{\mathbf{q}}\right) & \times \phi\left(\mathbf{n}_{\mathbf{q}}\right) \times \nabla_{\mathbf{t}_{\mathbf{q}}}^{2} \phi\left(\mathbf{n}_{\mathbf{q}}\right)=-\left([ \varkappa _ { 1 } ^ { 2 } + \varkappa _ { 3 } \varpi ] \varkappa _ { 1 } \left[\frac{\partial}{\partial s}\left\{\varkappa_{1}^{2}+\varkappa_{3} \varpi\right\}+\left\{\frac{\partial \varkappa_{1}}{\partial s}+\varkappa_{2} \varpi\right\} \varkappa_{1}\right.\right. \\
& \left.+\varkappa_{3}\left\{\frac{\partial \varpi}{\partial s}-\varkappa_{1} \varkappa_{2}\right\}\right]+\left[\varpi^{\prime}-\varkappa_{1} \varkappa_{2}\right]\left[\varkappa _ { 1 } \left\{\frac{\partial}{\partial s}\left(\frac{\partial \varpi}{\partial s}-\varkappa_{1} \varkappa_{2}\right)-\left(\frac{\partial \varkappa_{1}}{\partial s}+\varkappa_{2} \varpi\right) \varkappa_{2}\right.\right. \\
& \left.\left.\left.-\left(\varkappa_{1}^{2}+\varkappa_{3} \varpi\right) \varkappa_{3}\right\}+\varpi\left\{\varkappa_{1}\left(\varkappa_{1}^{2}+\varkappa_{3} \varpi\right)-\frac{\partial}{\partial s}\left(\frac{\partial \varkappa_{1}}{\partial s}+\varkappa_{2} \varpi\right)-\varkappa_{2}\left(\frac{\partial \varpi}{\partial s}-\varkappa_{1} \varkappa_{2}\right)\right\}\right]\right) \mathbf{t}_{\mathbf{q}} \\
& +\left(\varkappa_{1}\left[\frac{\partial}{\partial s}\left\{\varkappa_{1}^{2}+\varkappa_{3} \varpi\right\}+\left\{\frac{\partial \varkappa_{1}}{\partial s}+\varkappa_{2} \varpi\right\} \varkappa_{1}+\varkappa_{3}\left\{\frac{\partial \varpi}{\partial s}-\varkappa_{1} \varkappa_{2}\right\}\right]\left[\varkappa_{1}^{\prime}+\varkappa_{2} \varpi\right]+\left[\varpi^{\prime}-\varkappa_{1} \varkappa_{2}\right]\right. \\
& \left.\times \varpi\left[\frac{\partial}{\partial s}\left\{\varkappa_{1}^{2}+\varkappa_{3} \varpi\right\}+\left\{\frac{\partial \varkappa_{1}}{\partial s}+\varkappa_{2} \varpi\right\} \varkappa_{1}+\varkappa_{3}\left\{\frac{\partial \varpi}{\partial s}-\varkappa_{1} \varkappa_{2}\right\}\right]\right) \mathbf{n}_{\mathbf{q}}+\left(\varpi \left[\frac{\partial}{\partial s}\left\{\varkappa_{1}^{2}+\varkappa_{3} \varpi\right\}\right.\right. \\
& \left.+\left\{\frac{\partial \varkappa_{1}}{\partial s}+\varkappa_{2} \varpi\right\} \varkappa_{1}+\varkappa_{3}\left\{\frac{\partial \varpi}{\partial s}-\varkappa_{1} \varkappa_{2}\right\}\right]\left[\varkappa_{1}^{2}+\varkappa_{3} \varpi\right]-\left[\varkappa_{1}^{\prime}+\varkappa_{2} \varpi\right]\left[\varkappa _ { 1 } \left\{\frac{\partial}{\partial s}\left(\frac{\partial \varpi}{\partial s}-\varkappa_{1} \varkappa_{2}\right)\right.\right. \\
& \left.\left.\left.-\left(\frac{\partial \varkappa_{1}}{\partial s}+\varkappa_{2} \varpi\right) \varkappa_{2}-\left(\varkappa_{1}^{2}+\varkappa_{3} \varpi\right) \varkappa_{3}\right\}+\varpi\left\{\varkappa_{1}\left(\varkappa_{1}^{2}+\varkappa_{3} \varpi\right)-\frac{\partial}{\partial s}\left(\frac{\partial \varkappa_{1}}{\partial s}+\varkappa_{2} \varpi\right)-\varkappa_{2}\left(\frac{\partial \varpi}{\partial s}-\varkappa_{1} \varkappa_{2}\right)\right\}\right]\right) \mathbf{b}_{\mathbf{q}} .
\end{aligned}
$$

Similarly, ferromagnetic magnetic $\phi\left(\mathbf{n}_{\mathbf{q}}\right)$ flux is given by

$$
\begin{aligned}
& { }^{m} \mathcal{F}_{\phi\left(\mathbf{n}_{\mathbf{q}}\right)}^{\mathfrak{f e r r o} \mathfrak{f}}=\int_{\mathcal{F}}\left(-\varpi\left[\{ \varkappa _ { 1 } ^ { 2 } + \varkappa _ { 3 } \varpi \} \varkappa _ { 1 } \left\{\frac{\partial}{\partial s}\left(\varkappa_{1}^{2}+\varkappa_{3} \varpi\right)+\left(\frac{\partial \varkappa_{1}}{\partial s}+\varkappa_{2} \varpi\right) \varkappa_{1}\right.\right.\right. \\
& \left.+\varkappa_{3}\left(\frac{\partial \varpi}{\partial s}-\varkappa_{1} \varkappa_{2}\right)\right\}+\left[\varpi^{\prime}-\varkappa_{1} \varkappa_{2}\right]\left[\varkappa _ { 1 } \left\{\frac{\partial}{\partial s}\left(\frac{\partial \varpi}{\partial s}-\varkappa_{1} \varkappa_{2}\right)\right.\right. \\
& \left.-\left(\frac{\partial \varkappa_{1}}{\partial s}+\varkappa_{2} \varpi\right) \varkappa_{2}-\left(\varkappa_{1}^{2}+\varkappa_{3} \varpi\right) \varkappa_{3}\right\}+\varpi\left\{\varkappa_{1}\left(\varkappa_{1}^{2}+\varkappa_{3} \varpi\right)-\frac{\partial}{\partial s}\left(\frac{\partial \varkappa_{1}}{\partial s}+\varkappa_{2} \varpi\right)\right. \\
& \left.\left.\left.-\varkappa_{2}\left(\frac{\partial \varpi}{\partial s}-\varkappa_{1} \varkappa_{2}\right)\right\}\right]\right)-\varkappa_{2}\left(\varkappa _ { 1 } \left[\frac{\partial}{\partial s}\left\{\varkappa_{1}^{2}+\varkappa_{3} \varpi\right\}+\left\{\frac{\partial \varkappa_{1}}{\partial s}+\varkappa_{2} \varpi\right\} \varkappa_{1}\right.\right. \\
& \left.+\varkappa_{3}\left\{\frac{\partial \varpi}{\partial s}-\varkappa_{1} \varkappa_{2}\right\}\right]\left[\varkappa_{1}^{\prime}+\varkappa_{2} \varpi\right]+\left[\varpi^{\prime}-\varkappa_{1} \varkappa_{2}\right] \varpi\left[\frac{\partial}{\partial s}\left\{\varkappa_{1}^{2}+\varkappa_{3} \varpi\right\}+\left\{\frac{\partial \varkappa_{1}}{\partial s}+\varkappa_{2} \varpi\right\} \varkappa_{1}\right. \\
& \left.\left.+\varkappa_{3}\left\{\frac{\partial \varpi}{\partial s}-\varkappa_{1} \varkappa_{2}\right\}\right]\right)+\varkappa_{1}\left(\varpi \left[\frac{\partial}{\partial s}\left\{\varkappa_{1}^{2}+\varkappa_{3} \varpi\right\}+\left\{\frac{\partial \varkappa_{1}}{\partial s}+\varkappa_{2} \varpi\right\} \varkappa_{1}\right.\right. \\
& \left.+\varkappa_{3}\left\{\frac{\partial \varpi}{\partial s}-\varkappa_{1} \varkappa_{2}\right\}\right]\left[\varkappa_{1}^{2}+\varkappa_{3} \varpi\right]-\left[\varkappa_{1}^{\prime}+\varkappa_{2} \varpi\right]\left[\varkappa _ { 1 } \left\{\frac{\partial}{\partial s}\left(\frac{\partial \varpi}{\partial s}-\varkappa_{1} \varkappa_{2}\right)\right.\right. \\
& \left.-\left(\frac{\partial \varkappa_{1}}{\partial s}+\varkappa_{2} \varpi\right) \varkappa_{2}-\left(\varkappa_{1}^{2}+\varkappa_{3}\right) \varkappa_{3}\right\}+\varpi\left\{\varkappa_{1}\left(\varkappa_{1}^{2}+\varkappa_{3} \varpi\right)-\frac{\partial}{\partial s}\left(\frac{\partial \varkappa_{1}}{\partial s}+\varkappa_{2} \varpi\right)\right. \\
& \left.\left.\left.-\varkappa_{2}\left(\frac{\partial \varpi}{\partial s}-\varkappa_{1} \varkappa_{2}\right)\right\}\right]\right) d \pi
\end{aligned}
$$


The condition of magnetic $\phi\left(\mathbf{n}_{\mathbf{q}}\right)$ flux surface is given by

$$
\begin{aligned}
\left(\left[\frac{\partial \varpi}{\partial s}\right.\right. & \left.-\varkappa_{1} \varkappa_{2}\right]\left[\varkappa_{1}\left\{\frac{\partial \beta_{2}}{\partial s}+\varkappa_{1} \beta_{1}-\varkappa_{3} \beta_{3}\right\}+\varpi \chi\right] \\
& \left.-\left[\varkappa_{1}^{2}+\varkappa_{3} \varpi\right]\left[\frac{\partial \varpi}{\partial t}-\varkappa_{1}\left\{\frac{\partial \beta_{3}}{\partial s}+\varkappa_{2} \beta_{1}+\varkappa_{3} \beta_{2}\right\}\right]\right) \varpi \\
& -\varkappa_{2}\left(\left[\frac{\partial \varkappa_{1}}{\partial s}+\varkappa_{2} \varpi\right]\left[\frac{\partial \varpi}{\partial t}-\varkappa_{1}\left\{\frac{\partial \beta_{3}}{\partial s}+\varkappa_{2} \beta_{1}+\varkappa_{3} \beta_{2}\right\}\right]\right. \\
& \left.-\left[\frac{\partial \varpi}{\partial s}-\varkappa_{1} \varkappa_{2}\right]\left[\frac{\partial \varkappa_{1}}{\partial t}+\varpi\left\{\frac{\partial \beta_{3}}{\partial s}+\varkappa_{2} \beta_{1}+\varkappa_{3} \beta_{2}\right\}\right]\right) \\
& +\varkappa_{1}\left(\left[\frac{\partial \varkappa_{1}}{\partial s}+\varkappa_{2} \varpi\right]\left[\varkappa_{1}\left\{\frac{\partial \beta_{2}}{\partial s}+\varkappa_{1} \beta_{1}-\varkappa_{3} \beta_{3}\right\}+\varpi \chi\right]\right. \\
& \left.-\left[\varkappa_{1}^{2}+\varkappa_{3} \varpi\right]\left[\frac{\partial \varkappa_{1}}{\partial t}+\varpi\left\{\frac{\partial \beta_{3}}{\partial s}+\varkappa_{2} \beta_{1}+\varkappa_{3} \beta_{2}\right\}\right]\right)=0 .
\end{aligned}
$$

The magnetic $\phi\left(\mathbf{n}_{\mathbf{q}}\right)$ flux surface is given by the following ferromagnetic condition

$$
\begin{aligned}
& -\varpi\left(\left[\varkappa_{1}^{2}+\varkappa_{3} \varpi\right] \varkappa_{1}\left[\frac{\partial}{\partial s}\left\{\varkappa_{1}^{2}+\varkappa_{3} \varpi\right\}+\left\{\frac{\partial \varkappa_{1}}{\partial s}+\varkappa_{2} \varpi\right\} \varkappa_{1}+\varkappa_{3}\left\{\frac{\partial \varpi}{\partial s}-\varkappa_{1} \varkappa_{2}\right\}\right]\right. \\
& +\left[\varpi^{\prime}-\varkappa_{1} \varkappa_{2}\right]\left[\varkappa_{1}\left\{\frac{\partial}{\partial s}\left(\frac{\partial \varpi}{\partial s}-\varkappa_{1} \varkappa_{2}\right)-\left(\frac{\partial \varkappa_{1}}{\partial s}+\varkappa_{2} \varpi\right) \varkappa_{2}-\left(\varkappa_{1}^{2}+\varkappa_{3} \varpi\right) \varkappa_{3}\right\}\right. \\
& \left.\left.+\varpi\left\{\varkappa_{1}\left(\varkappa_{1}^{2}+\varkappa_{3} \varpi\right)-\frac{\partial}{\partial s}\left(\frac{\partial \varkappa_{1}}{\partial s}+\varkappa_{2} \varpi\right)-\varkappa_{2}\left(\frac{\partial \varpi}{\partial s}-\varkappa_{1} \varkappa_{2}\right)\right\}\right]\right) \\
& -\varkappa_{2}\left(\varkappa_{1}\left[\frac{\partial}{\partial s}\left\{\varkappa_{1}^{2}+\varkappa_{3} \varpi\right\}+\left\{\frac{\partial \varkappa_{1}}{\partial s}+\varkappa_{2} \varpi\right\} \varkappa_{1}+\varkappa_{3}\left\{\frac{\partial \varpi}{\partial s}-\varkappa_{1} \varkappa_{2}\right\}\right]\left[\varkappa_{1}^{\prime}+\varkappa_{2} \varpi\right]\right. \\
& \left.+\left[\varpi^{\prime}-\varkappa_{1} \varkappa_{2}\right] \varpi\left[\frac{\partial}{\partial s}\left\{\varkappa_{1}^{2}+\varkappa_{3} \varpi\right\}+\left\{\frac{\partial \varkappa_{1}}{\partial s}+\varkappa_{2} \varpi\right\} \varkappa_{1}+\varkappa_{3}\left\{\frac{\partial \varpi}{\partial s}-\varkappa_{1} \varkappa_{2}\right\}\right]\right) \\
& +\varkappa_{1}\left(\varpi\left[\frac{\partial}{\partial s}\left\{\varkappa_{1}^{2}+\varkappa_{3} \varpi\right\}+\left\{\frac{\partial \varkappa_{1}}{\partial s}+\varkappa_{2} \varpi\right\} \varkappa_{1}+\varkappa_{3}\left\{\frac{\partial \varpi}{\partial s}-\varkappa_{1} \varkappa_{2}\right\}\right]\left[\varkappa_{1}^{2}+\varkappa_{3} \varpi\right]\right. \\
& -\left[\varkappa_{1}^{\prime}+\varkappa_{2} \varpi\right]\left[\varkappa_{1}\left\{\frac{\partial}{\partial s}\left(\frac{\partial \varpi}{\partial s}-\varkappa_{1} \varkappa_{2}\right)-\left(\frac{\partial \varkappa_{1}}{\partial s}+\varkappa_{2} \varpi\right) \varkappa_{2}-\left(\varkappa_{1}^{2}+\varkappa_{3} \varpi\right) \varkappa_{3}\right\}\right. \\
& \left.\left.+\varpi\left\{\varkappa_{1}\left(\varkappa_{1}^{2}+\varkappa_{3} \varpi\right)-\frac{\partial}{\partial s}\left(\frac{\partial \varkappa_{1}}{\partial s}+\varkappa_{2} \varpi\right)-\varkappa_{2}\left(\frac{\partial \varpi}{\partial s}-\varkappa_{1} \varkappa_{2}\right)\right\}\right)\right]=0 .
\end{aligned}
$$

We consider the similar method as in the first case to extract the following demonstration. In Fig. 2, the magnetic flux density of the particle is shown when it is assumed under the action of the Lorentz force $\phi\left(\mathbf{n}_{\mathbf{q}}\right)$. 


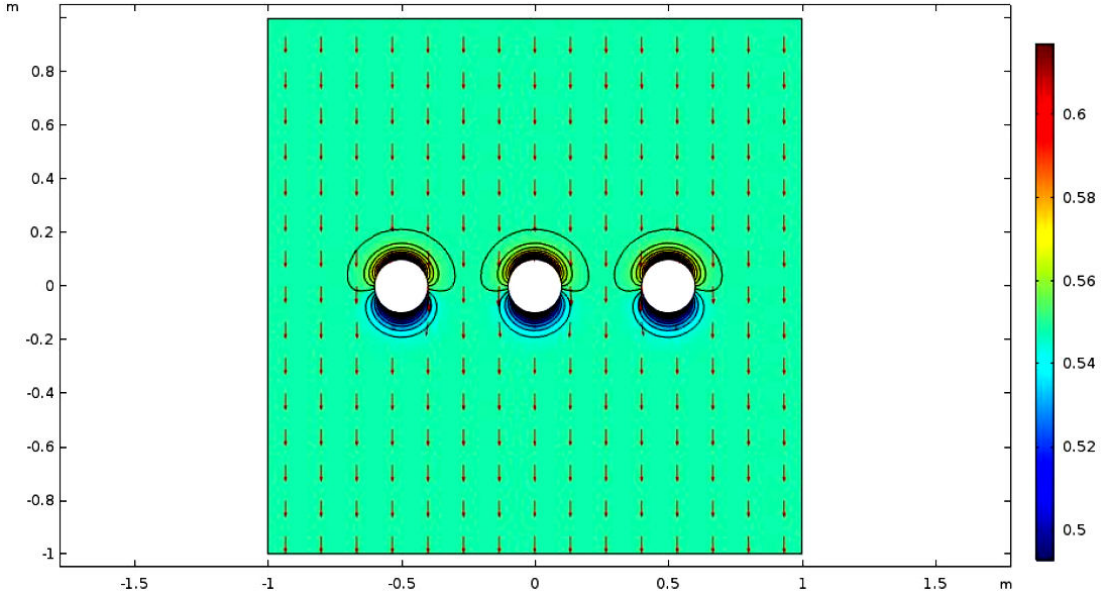

FIGURE 2. The magnetic flux density with Lorentz force $\phi\left(\mathbf{n}_{\mathbf{q}}\right)$.

\section{Case 3. Magnetic $\phi\left(\mathbf{b}_{\mathbf{q}}\right)$ flux}

The magnetic $\phi\left(\mathbf{b}_{\mathbf{q}}\right)$ flux ${ }^{m} \mathcal{F}_{\phi\left(\mathbf{b}_{\mathbf{q}}\right)}$ is given by

$$
\begin{aligned}
m_{\mathcal{F}_{\phi\left(\mathbf{b}_{\mathbf{q}}\right)}} & =\int_{\mathcal{F}}\left(\varpi \left[\left\{\frac{\partial \varpi}{\partial s}+\varkappa_{2} \varkappa_{1}\right\}\left\{\varkappa_{2}\left(\frac{\partial \beta_{3}}{\partial s}+\varkappa_{2} \beta_{1}+\varkappa_{3} \beta_{2}\right)+\varpi \chi\right\}\right.\right. \\
& \left.\left.-\left\{\varkappa_{2}^{2}+\varpi \varkappa_{3}\right\}\left\{\frac{\partial \varpi}{\partial t}+\varkappa_{2}\left(\frac{\partial \beta_{2}}{\partial s}+\varkappa_{1} \beta_{1}-\varkappa_{3} \beta_{3}\right)\right\}\right]\right] \\
& -\varkappa_{2}\left[\left\{-\frac{\partial \varkappa_{2}}{\partial s}+\varpi \varkappa_{1}\right\}\left\{\varkappa_{2}\left(\frac{\partial \beta_{3}}{\partial s}+\varkappa_{2} \beta_{1}+\varkappa_{3} \beta_{2}\right)+\varpi \chi\right\}\right. \\
& \left.\left.-\left\{\varkappa_{2}^{2}+\varpi \varkappa_{3}\right\}\left\{\varpi\left(\frac{\partial \beta_{2}}{\partial s}+\varkappa_{1} \beta_{1}-\varkappa_{3} \beta_{3}\right)-\frac{\partial \varkappa_{2}}{\partial t}\right\}\right]\right] \\
& +\varkappa_{1}\left[\left\{\frac{\partial \varpi}{\partial s}+\varkappa_{2} \varkappa_{1}\right\}\left\{\varpi\left(\frac{\partial \beta_{2}}{\partial s}+\varkappa_{1} \beta_{1}-\varkappa_{3} \beta_{3}\right)-\frac{\partial \varkappa_{2}}{\partial t}\right\}\right. \\
& \left.\left.-\left\{\frac{\partial \varpi}{\partial t}+\varkappa_{2}\left(\frac{\partial \beta_{2}}{\partial s}+\varkappa_{1} \beta_{1}-\varkappa_{3} \beta_{3}\right)\right\}\left\{-\frac{\partial \varkappa_{2}}{\partial s}+\varpi \varkappa_{1}\right\}\right]\right) d \pi .
\end{aligned}
$$

With short calculations, we have

$$
\begin{aligned}
\nabla_{s} \phi\left(\mathbf{b}_{\mathbf{q}}\right) \times \nabla_{t} \phi\left(\mathbf{b}_{\mathbf{q}}\right) & =\left(\left[\frac{\partial \varpi}{\partial s}+\varkappa_{2} \varkappa_{1}\right]\left[\varkappa_{2}\left\{\frac{\partial \beta_{3}}{\partial s}+\varkappa_{2} \beta_{1}+\varkappa_{3} \beta_{2}\right\}+\varpi \chi\right]-\left[\varkappa_{2}^{2}+\varpi \varkappa_{3}\right]\right. \\
& \left.\times\left[\frac{\partial \varpi}{\partial t}+\varkappa_{2}\left\{\frac{\partial \beta_{2}}{\partial s}+\varkappa_{1} \beta_{1}-\varkappa_{3} \beta_{3}\right\}\right]\right) \mathbf{t}_{\mathbf{q}}+\left([ - \frac { \partial \varkappa _ { 2 } } { \partial s } + \varpi \varkappa _ { 1 } ] \left[\varkappa _ { 2 } \left\{\frac{\partial \beta_{3}}{\partial s}\right.\right.\right. \\
& \left.\left.+\varkappa_{2} \beta_{1}+\varkappa_{3} \beta_{2}\right\}+\varpi \chi\right]-\left[\varkappa_{2}^{2}+\varpi \varkappa_{3}\right]\left[\varpi\left\{\frac{\partial \beta_{2}}{\partial s}+\varkappa_{1} \beta_{1}-\varkappa_{3} \beta_{3}\right\}\right. \\
& \left.\left.-\frac{\partial \varkappa_{2}}{\partial t}\right]\right) \mathbf{n}_{\mathbf{q}}+\left(\left[\frac{\partial \varpi}{\partial s}+\varkappa_{2} \varkappa_{1}\right]\left[\varpi\left\{\frac{\partial \beta_{2}}{\partial s}+\varkappa_{1} \beta_{1}-\varkappa_{3} \beta_{3}\right\}-\frac{\partial \varkappa_{2}}{\partial t}\right]\right. \\
& \left.-\left[\frac{\partial \varpi}{\partial t}+\varkappa_{2}\left\{\frac{\partial \beta_{2}}{\partial s}+\varkappa_{1} \beta_{1}-\varkappa_{3} \beta_{3}\right\}\right]\left[-\frac{\partial \varkappa_{2}}{\partial s}+\varpi \varkappa_{1}\right]\right) \mathbf{b}_{\mathbf{q}} .
\end{aligned}
$$


Flux density of $\phi\left(\mathbf{b}_{\mathbf{q}}\right)$ is given by

$$
\begin{aligned}
{ }^{m} \mathbf{L}_{\phi\left(\mathbf{b}_{\mathbf{q}}\right)} & =\varpi\left(\left[\frac{\partial \varpi}{\partial s}+\varkappa_{2} \varkappa_{1}\right]\left[\varkappa_{2}\left\{\frac{\partial \beta_{3}}{\partial s}+\varkappa_{2} \beta_{1}+\varkappa_{3} \beta_{2}\right\}+\varpi \chi\right]-\left[\varkappa_{2}^{2}+\varpi \varkappa_{3}\right]\left[\frac{\partial \varpi}{\partial t}+\varkappa_{2}\left\{\frac{\partial \beta_{2}}{\partial s}\right.\right.\right. \\
& \left.\left.\left.+\varkappa_{1} \beta_{1}-\varkappa_{3} \beta_{3}\right\}\right]\right)-\varkappa_{2}\left(\left[-\frac{\partial \varkappa_{2}}{\partial s}+\varpi \varkappa_{1}\right]\left[\varkappa_{2}\left\{\frac{\partial \beta_{3}}{\partial s}+\varkappa_{2} \beta_{1}+\varkappa_{3} \beta_{2}\right\}+\varpi \chi\right]\right. \\
& \left.-\left[\varkappa_{2}^{2}+\varpi \varkappa_{3}\right]\left[\varpi\left\{\frac{\partial \beta_{2}}{\partial s}+\varkappa_{1} \beta_{1}-\varkappa_{3} \beta_{3}\right\}-\frac{\partial \varkappa_{2}}{\partial t}\right]\right)+\varkappa_{1}\left([ \frac { \partial \varpi } { \partial s } + \varkappa _ { 2 } \varkappa _ { 1 } ] \left[\varpi \left\{\frac{\partial \beta_{2}}{\partial s}\right.\right.\right. \\
& \left.\left.\left.+\varkappa_{1} \beta_{1}-\varkappa_{3} \beta_{3}\right\}-\frac{\partial \varkappa_{2}}{\partial t}\right]-\left[\frac{\partial \varpi}{\partial t}+\varkappa_{2}\left\{\frac{\partial \beta_{2}}{\partial s}+\varkappa_{1} \beta_{1}-\varkappa_{3} \beta_{3}\right\}\right]\left[-\frac{\partial \varkappa_{2}}{\partial s}+\varpi \varkappa_{1}\right]\right) .
\end{aligned}
$$

Using the above equation in phase, we obtain that

$$
\begin{aligned}
m_{\mathcal{F}_{\phi\left(\mathbf{b}_{\mathbf{q}}\right)}} & =\int_{\mathcal{F}}\left(\varpi \left[\left\{\frac{\partial \varpi}{\partial s}+\varkappa_{2} \varkappa_{1}\right\}\left\{\varkappa_{2}\left(\frac{\partial \beta_{3}}{\partial s}+\varkappa_{2} \beta_{1}+\varkappa_{3} \beta_{2}\right)+\varpi \chi\right\}-\left\{\varkappa_{2}^{2}+\varpi \varkappa_{3}\right\}\left\{\frac{\partial \varpi}{\partial t}\right.\right.\right. \\
& \left.\left.+\varkappa_{2}\left(\frac{\partial \beta_{2}}{\partial s}+\varkappa_{1} \beta_{1}-\varkappa_{3} \beta_{3}\right)\right\}\right]-\varkappa_{2}\left[\left\{-\frac{\partial \varkappa_{2}}{\partial s}+\varpi \varkappa_{1}\right\}\left\{\varkappa_{2}\left(\frac{\partial \beta_{3}}{\partial s}+\varkappa_{2} \beta_{1}+\varkappa_{3} \beta_{2}\right)\right)\right. \\
& \left.+\varpi \chi\}-\left\{\varkappa_{2}^{2}+\varpi \varkappa_{3}\right\}\left\{\varpi\left(\frac{\partial \beta_{2}}{\partial s}+\varkappa_{1} \beta_{1}-\varkappa_{3} \beta_{3}\right)-\frac{\partial \varkappa_{2}}{\partial t}\right\}\right]+\varkappa_{1}\left[\{ \frac { \partial \varpi } { \partial s } + \varkappa _ { 2 } \varkappa _ { 1 } \} \left\{\varpi \left(\frac{\partial \beta_{2}}{\partial s}\right.\right.\right. \\
& \left.\left.\left.\left.+\varkappa_{1} \beta_{1}-\varkappa_{3} \beta_{3}\right)-\frac{\partial \varkappa_{2}}{\partial t}\right\}-\left\{\frac{\partial \varpi}{\partial t}+\varkappa_{2}\left(\frac{\partial \beta_{2}}{\partial s}+\varkappa_{1} \beta_{1}-\varkappa_{3} \beta_{3}\right)\right\}\left\{-\frac{\partial \varkappa_{2}}{\partial s}+\varpi \varkappa_{1}\right\}\right]\right) d \pi .
\end{aligned}
$$

Also, ferromagnetic model for $\phi\left(\mathbf{b}_{\mathbf{q}}\right)$, we get that

$$
{ }^{m} \mathbf{L}_{\phi\left(\mathbf{b}_{\mathbf{q}}\right)}^{\mathfrak{f e r r o}}=\mathcal{B} \cdot \nabla_{s} \phi\left(\mathbf{b}_{\mathbf{q}}\right) \times \phi\left(\mathbf{b}_{\mathbf{q}}\right) \times \nabla_{s}^{2} \phi\left(\mathbf{b}_{\mathbf{q}}\right) .
$$

By using the quasi frame, we have

$$
\begin{aligned}
\nabla_{s} \phi\left(\mathbf{b}_{\mathbf{q}}\right) & \times \phi\left(\mathbf{b}_{\mathbf{q}}\right) \times \nabla_{\mathbf{t}_{\mathbf{q}}}^{2} \phi\left(\mathbf{b}_{\mathbf{q}}\right)=\left(\varkappa _ { 2 } \left[\varkappa_{2}\left\{-\frac{\partial \varkappa_{2}}{\partial s}+\varpi \varkappa_{1}\right\}-\left\{\frac{\partial \varpi}{\partial s}+\varkappa_{2} \varkappa_{1}\right\} \varkappa_{3}\right.\right. \\
& \left.-\frac{\partial}{\partial s}\left\{\varkappa_{2}^{2}+\varpi \varkappa_{3}\right\}\right]\left[\varkappa_{2}^{2}+\varpi \varkappa_{3}\right]-\left[\varpi^{\prime}+\varkappa_{2} \varkappa_{1}\right]\left[\begin{array}{l}
\partial \\
\partial s
\end{array}-\frac{\partial \varkappa_{2}}{\partial s}+\varpi \varkappa_{1}\right)+\left(\frac{\partial \varpi}{\partial s}+\varkappa_{2} \varkappa_{1}\right) \varkappa_{1} \\
& \left.\left.\left.+\left(\varkappa_{2}^{2}+\varpi \varkappa_{3}\right) \varkappa_{2}\right\} \varkappa_{2}\left\{\left(-\frac{\partial \varkappa_{2}}{\partial s}+\varpi \varkappa_{1}\right) \varkappa_{1}-\frac{\partial}{\partial s}\left(\frac{\partial \varpi}{\partial s}+\varkappa_{2} \varkappa_{1}\right)+\left(\varkappa_{2}^{2}+\varpi \varkappa_{3}\right) \varkappa_{3}\right\}\right]\right) \mathbf{t}_{\mathbf{q}} \\
& -\left([ \varpi \varkappa _ { 1 } - \varkappa _ { 2 } ^ { \prime } ] \left[\varpi\left\{\frac{\partial}{\partial s}\left(-\frac{\partial \varkappa_{2}}{\partial s}+\varpi \varkappa_{1}\right)+\left(\frac{\partial \varpi}{\partial s}+\varkappa_{2} \varkappa_{1}\right) \varkappa_{1}+\left(\varkappa_{2}^{2}+\varpi \varkappa_{3}\right) \varkappa_{2}\right\}\right.\right. \\
& \left.\left.-\varkappa_{2}\left\{\left(-\frac{\partial \varkappa_{2}}{\partial s}+\varpi \varkappa_{1}\right) \varkappa_{1}-\frac{\partial}{\partial s} \frac{\partial \varpi s}{\partial s}+\varkappa_{2} \varkappa_{1}\right\}+\left\{\varkappa_{2}^{2}+\varpi \varkappa_{3}\right\} \varkappa_{3}\right]\right) \\
& +\left(\varkappa_{2}^{2}+\varpi \varkappa_{3}\right) \varpi\left(\varkappa_{2}\left[-\frac{\partial \varkappa_{2}}{\partial s}+\varpi \varkappa_{1}\right]-\left[\frac{\partial \varpi}{\partial s}+\varkappa_{2} \varkappa_{1}\right] \varkappa_{3}-\frac{\partial}{\partial s}\left[\varkappa_{2}^{2}+\varpi \varkappa_{3}\right]\right) \mathbf{n}_{\mathbf{q}} \\
& \left.+\left[\varpi^{\prime}+\varkappa_{2} \varkappa_{1}\right] \varpi\left[\varkappa_{2}\left\{-\frac{\partial \varkappa_{2}}{\partial s}+\varpi \varkappa_{1}\right\}-\left\{\frac{\partial \varpi}{\partial s}+\varkappa_{2} \varkappa_{1}\right\} \varkappa_{3}-\frac{\partial}{\partial s}\left\{\varkappa_{2}^{2}+\varpi \varkappa_{3}\right\}\right]\right) \mathbf{b}_{\mathbf{q}} . \\
& \left(\left[\varkappa_{2}\left\{-\frac{\partial \varkappa_{2}}{\partial s}+\varpi \varkappa_{1}\right\}-\left\{\frac{\partial \varpi}{\partial s}+\varkappa_{2} \varkappa_{1}\right\} \varkappa_{3}-\frac{\partial}{\partial s}\left\{\varkappa_{2}^{2}+\varpi \varkappa_{3}\right\}\right]\right.
\end{aligned}
$$


Also, we find that

$$
\begin{aligned}
{ }^{m} \mathbf{L}_{\phi\left(\mathbf{b}_{\mathbf{q}}\right)}^{\mathrm{ferro}} & =\varpi\left(\varkappa_{2}\left[\varkappa_{2}\left\{-\frac{\partial \varkappa_{2}}{\partial s}+\varpi \varkappa_{1}\right\}-\left\{\frac{\partial \varpi}{\partial s}+\varkappa_{2} \varkappa_{1}\right\} \varkappa_{3}-\frac{\partial}{\partial s}\left\{\varkappa_{2}^{2}+\varpi \varkappa_{3}\right\}\right]\left[\varkappa_{2}^{2}+\varpi \varkappa_{3}\right]\right. \\
& -\left[\varpi^{\prime}+\varkappa_{2} \varkappa_{1}\right]\left[\varpi\left\{\frac{\partial}{\partial s}\left(-\frac{\partial \varkappa_{2}}{\partial s}+\varpi \varkappa_{1}\right)+\left(\frac{\partial \varpi}{\partial s}+\varkappa_{2} \varkappa_{1}\right) \varkappa_{1}+\left(\varkappa_{2}^{2}+\varpi \varkappa_{3}\right) \varkappa_{2}\right\}\right. \\
& \left.\left.-\varkappa_{2}\left\{\left(-\frac{\partial \varkappa_{2}}{\partial s}+\varpi \varkappa_{1}\right) \varkappa_{1}-\frac{\partial}{\partial s}\left(\frac{\partial \varpi}{\partial s}+\varkappa_{2} \varkappa_{1}\right)+\left(\varkappa_{2}^{2}+\varpi \varkappa_{3}\right) \varkappa_{3}\right\}\right]\right)+\varkappa_{1}\left(\left[\varpi \varkappa_{1}-\varkappa_{2}^{\prime}\right] \varkappa_{2}\right. \\
& \times\left[\varkappa_{2}\left\{-\frac{\partial \varkappa_{2}}{\partial s}+\varpi \varkappa_{1}\right\}-\left\{\frac{\partial \varpi}{\partial s}+\varkappa_{2} \varkappa_{1}\right\} \varkappa_{3}-\frac{\partial}{\partial s}\left\{\varkappa_{2}^{2}+\varpi \varkappa_{3}\right\}\right]-\left[\varpi^{\prime}+\varkappa_{2} \varkappa_{1}\right] \varpi\left[\varkappa_{2}\right. \\
& \left.\left.\times\left\{-\frac{\partial \varkappa_{2}}{\partial s}+\varpi \varkappa_{1}\right\}-\left\{\frac{\partial \varpi}{\partial s}+\varkappa_{2} \varkappa_{1}\right\} \varkappa_{3}-\frac{\partial}{\partial s}\left\{\varkappa_{2}^{2}+\varpi \varkappa_{3}\right\}\right]\right)+\varkappa_{2}\left([ \varpi \varkappa _ { 1 } - \varkappa _ { 2 } ^ { \prime } ] \left[\varpi \left\{\frac{\partial}{\partial s}\right.\right.\right. \\
& \left.\times\left(-\frac{\partial \varkappa_{2}}{\partial s}+\varpi \varkappa_{1}\right)+\left(\frac{\partial \varpi}{\partial s}+\varkappa_{2} \varkappa_{1}\right) \varkappa_{1}+\left(\varkappa_{2}^{2}+\varpi \varkappa_{3}\right) \varkappa_{2}\right\}-\varkappa_{2}\left\{\left(-\frac{\partial \varkappa_{2}}{\partial s}+\varpi \varkappa_{1}\right) \varkappa_{1}\right. \\
& \left.\left.-\frac{\partial}{\partial s}\left(\frac{\partial \varpi}{\partial s}+\varkappa_{2} \varkappa_{1}\right)+\left(\varkappa_{2}^{2}+\varpi \varkappa_{3}\right) \varkappa_{3}\right\}\right]+\left[\varkappa_{2}^{2}+\varpi \varkappa_{3}\right] \varpi\left[\varkappa_{2}\left\{-\frac{\partial \varkappa_{2}}{\partial s}+\varpi \varkappa_{1}\right\}\right. \\
& \left.\left.-\left\{\frac{\partial \varpi}{\partial s}+\varkappa_{2} \varkappa_{1}\right\} \varkappa_{3}-\frac{\partial}{\partial s}\left\{\varkappa_{2}^{2}+\varpi \varkappa_{3}\right\}\right]\right) .
\end{aligned}
$$

Thus, we immediately obtain that

$$
\begin{aligned}
& { }^{m} \mathcal{F}_{\phi\left(\mathbf{b}_{\mathbf{q}}\right)}^{\mathfrak{f e r r o}}=\int_{\mathcal{F}}\left(\varpi \left[\varkappa_{2}\left\{\varkappa_{2}\left(-\frac{\partial \varkappa_{2}}{\partial s}+\varpi \varkappa_{1}\right)-\left(\frac{\partial \varpi}{\partial s}+\varkappa_{2} \varkappa_{1}\right)\right) \varkappa_{3}\right.\right. \\
& \left.-\frac{\partial}{\partial s}\left(\varkappa_{2}^{2}+\varpi \varkappa_{3}\right)\right\}\left\{\varkappa_{2}^{2}+\varpi \varkappa_{3}\right\}-\left\{\varpi^{\prime}+\varkappa_{2} \varkappa_{1}\right\}\left\{\varpi \left(\frac{\partial}{\partial s}\left[-\frac{\partial \varkappa_{2}}{\partial s}+\varpi \varkappa_{1}\right]\right.\right. \\
& \left.+\left\{\frac{\partial \varpi}{\partial s}+\varkappa_{2} \varkappa_{1}\right]-\varkappa_{1}+\left[\varkappa_{2}^{2}+\varpi \varkappa_{3}\right] \varkappa_{2}\right)-\varkappa_{2}\left(\left[-\frac{\partial \varkappa_{2}}{\partial s}+\varpi \varkappa_{1}\right]-\varkappa_{1}\right. \\
& \left.\left.\left.-\frac{\partial}{\partial s}\left\{\frac{\partial \varpi}{\partial s}+\varkappa_{2} \varkappa_{1}\right]+\left\{\varkappa_{2}^{2}+\varpi \varkappa_{3}\right] \varkappa_{3}\right)\right\}\right]+\varkappa_{1}\left[\left\{\varpi \varkappa_{1}-\varkappa_{2}^{\prime}\right\} \varkappa_{2}\right. \\
& \times\left\{\varkappa_{2}\left(-\frac{\partial \varkappa_{2}}{\partial s}+\varpi \varkappa_{1}\right)-\left(\frac{\partial \varpi}{\partial s}+\varkappa_{2} \varkappa_{1}\right) \varkappa_{3}-\frac{\partial}{\partial s}\left(\varkappa_{2}^{2}+\varpi \varkappa_{3}\right)\right\} \\
& -\left\{\varpi^{\prime}+\varkappa_{2} \varkappa_{1}\right\} \varpi\left\{\varkappa_{2}\left(-\frac{\partial \varkappa_{2}}{\partial s}+\varpi \varkappa_{1}\right)-\left(\frac{\partial \varpi}{\partial s}+\varkappa_{2} \varkappa_{1}\right) \varkappa_{3}\right. \\
& \left.\left.-\frac{\partial}{\partial s}\left(\varkappa_{2}^{2}+\varpi \varkappa_{3}\right)\right\}\right]+\varkappa_{2}\left[\{ \varpi \varkappa _ { 1 } - \varkappa _ { 2 } ^ { \prime } \} \left\{\varpi \left(\frac{\partial}{\partial s}\left\{-\frac{\partial \varkappa_{2}}{\partial s}+\varpi \varkappa_{1}\right]\right.\right.\right. \\
& \left.+\left[\frac{\partial \varpi}{\partial s}+\varkappa_{2} \varkappa_{1}\right]-\varkappa_{1}+\left[\varkappa_{2}^{2}+\varpi \varkappa_{3}\right] \varkappa_{2}\right)-\varkappa_{2}\left(\left[-\frac{\partial \varkappa_{2}}{\partial s}+\varpi \varkappa_{1}\right]-\varkappa_{1}\right. \\
& \left.\left.-\frac{\partial}{\partial s}\left[\frac{\partial \varpi}{\partial s}+\varkappa_{2} \varkappa_{1}\right]+\left\{\varkappa_{2}^{2}+\varpi \varkappa_{3}\right\} \varkappa_{3}\right)\right\}+\left\{\varkappa_{2}^{2}+\varpi \varkappa_{3}\right\} \varpi\left\{\varkappa_{2}\right. \\
& \left.\left.\left.\times\left(-\frac{\partial \varkappa_{2}}{\partial s}+\varpi \varkappa_{1}\right)-\left(\frac{\partial \varpi}{\partial s}+\varkappa_{2} \varkappa_{1}\right) \varkappa_{3}-\frac{\partial}{\partial s}\left(\varkappa_{2}^{2}+\varpi \varkappa_{3}\right)\right\}\right]\right) d \pi .
\end{aligned}
$$


The magnetic $\phi\left(\mathbf{b}_{\mathbf{q}}\right)$ flux surface condition is given by

$$
\begin{aligned}
& \varpi\left(\left[\frac{\partial \varpi}{\partial s}+\varkappa_{2} \varkappa_{1}\right]\left[\varkappa_{2}\left\{\frac{\partial \beta_{3}}{\partial s}+\varkappa_{2} \beta_{1}+\varkappa_{3} \beta_{2}\right\}+\varpi \chi\right]\right. \\
& \left.-\left[\varkappa_{2}^{2}+\varpi \varkappa_{3}\right]\left[\frac{\partial \varpi}{\partial t}+\varkappa_{2}\left\{\frac{\partial \beta_{2}}{\partial s}+\varkappa_{1} \beta_{1}-\varkappa_{3} \beta_{3}\right\}\right]\right) \\
& -\varkappa_{2}\left(\left[-\frac{\partial \varkappa_{2}}{\partial s}+\varpi \varkappa_{1}\right]\left[\varkappa_{2}\left\{\frac{\partial \beta_{3}}{\partial s}+\varkappa_{2} \beta_{1}+\varkappa_{3} \beta_{2}\right\}+\varpi \chi\right]\right. \\
& \left.-\left[\varkappa_{2}^{2}+\varpi \varkappa_{3}\right]\left[\varpi\left\{\frac{\partial \beta_{2}}{\partial s}+\varkappa_{1} \beta_{1}-\varkappa_{3} \beta_{3}\right\}-\frac{\partial \varkappa_{2}}{\partial t}\right]\right) \\
& +\varkappa_{1}\left(\left[\frac{\partial \varpi}{\partial s}+\varkappa_{2} \varkappa_{1}\right]\left[\varpi\left\{\frac{\partial \beta_{2}}{\partial s}+\varkappa_{1} \beta_{1}-\varkappa_{3} \beta_{3}\right\}-\frac{\partial \varkappa_{2}}{\partial t}\right]\right. \\
& \left.-\left[\frac{\partial \varpi}{\partial t}+\varkappa_{2}\left\{\frac{\partial \beta_{2}}{\partial s}+\varkappa_{1} \beta_{1}-\varkappa_{3} \beta_{3}\right\}\right]\left[-\frac{\partial \varkappa_{2}}{\partial s}+\varpi \varkappa_{1}\right]\right)=0 .
\end{aligned}
$$

The magnetic $\phi\left(\mathbf{b}_{\mathbf{q}}\right)$ flux surface is given by the ferromagnetic condition

$$
\begin{aligned}
& \varpi\left(\varkappa_{2}\left[\varkappa_{2}\left\{-\frac{\partial \varkappa_{2}}{\partial s}+\varpi \varkappa_{1}\right\}-\left\{\frac{\partial \varpi}{\partial s}+\varkappa_{2} \varkappa_{1}\right\} \varkappa_{3}-\frac{\partial}{\partial s}\left\{\varkappa_{2}^{2}+\varpi \varkappa_{3}\right\}\right]\left[\varkappa_{2}^{2}+\varpi \varkappa_{3}\right]\right. \\
& -\left[\varpi^{\prime}+\varkappa_{2} \varkappa_{1}\right]\left[\varpi\left\{\frac{\partial}{\partial s}\left(-\frac{\partial \varkappa_{2}}{\partial s}+\varpi \varkappa_{1}\right)+\left(\frac{\partial \varpi}{\partial s}+\varkappa_{2} \varkappa_{1}\right) \varkappa_{1}+\left(\varkappa_{2}^{2}+\varpi \varkappa_{3}\right) \varkappa_{2}\right\}\right. \\
& \left.\left.-\varkappa_{2}\left\{\left(-\frac{\partial \varkappa_{2}}{\partial s}+\varpi \varkappa_{1}\right) \varkappa_{1}-\frac{\partial}{\partial s}\left(\frac{\partial \varpi}{\partial s}+\varkappa_{2} \varkappa_{1}\right)+\left(\varkappa_{2}^{2}+\varpi \varkappa_{3}\right) \varkappa_{3}\right\}\right]\right)+\varkappa_{1}\left(\left[\varpi \varkappa_{1}-\varkappa_{2}^{\prime}\right] \varkappa_{2}\right. \\
& \times\left[\varkappa_{2}\left\{-\frac{\partial \varkappa_{2}}{\partial s}+\varpi \varkappa_{1}\right\}-\left\{\frac{\partial \varpi}{\partial s}+\varkappa_{2} \varkappa_{1}\right\} \varkappa_{3}-\frac{\partial}{\partial s}\left\{\varkappa_{2}^{2}+\varpi \varkappa_{3}\right\}\right]-\left[\varpi^{\prime}+\varkappa_{2} \varkappa_{1}\right] \varpi\left[\varkappa_{2}\left\{-\frac{\partial \varkappa_{2}}{\partial s}+\varpi \varkappa_{1}\right\}\right. \\
& \left.-\left\{\frac{\partial \varpi}{\partial s}+\varkappa_{2} \varkappa_{1}\right\} \varkappa_{3}-\frac{\partial}{\partial s}\left\{\varkappa_{2}^{2}+\varpi \varkappa_{3}\right\}\right]+\varkappa_{2}\left([ \varpi \varkappa _ { 1 } - \varkappa _ { 2 } ^ { \prime } ] \left[\varpi \left\{\frac{\partial}{\partial s}\left(-\frac{\partial \varkappa_{2}}{\partial s}+\varpi \varkappa_{1}\right)\right.\right.\right. \\
& \left.+\left(\frac{\partial \varpi}{\partial s}+\varkappa_{2} \varkappa_{1}\right) \varkappa_{1}+\left(\varkappa_{2}^{2}+\varpi \varkappa_{3}\right) \varkappa_{2}\right\}-\varkappa_{2}\left\{\left(-\frac{\partial \varkappa_{2}}{\partial s}+\varpi \varkappa_{1}\right) \varkappa_{1}-\frac{\partial}{\partial s}\left(\frac{\partial \varpi}{\partial s}+\varkappa_{2} \varkappa_{1}\right)\right. \\
& \left.\left.\left.+\left(\varkappa_{2}^{2}+\varpi \varkappa_{3}\right) \varkappa_{3}\right\}\right]+\left[\varkappa_{2}^{2}+\varpi \varkappa_{3}\right] \varpi\left[\varkappa_{2}\left\{-\frac{\partial \varkappa_{2}}{\partial s}+\varpi \varkappa_{1}\right\}-\left\{\frac{\partial \varpi}{\partial s}+\varkappa_{2} \varkappa_{1}\right\} \varkappa_{3}-\frac{\partial}{\partial s}\left\{\varkappa_{2}^{2}+\varpi \varkappa_{3}\right\}\right]\right)=0 .
\end{aligned}
$$

We consider the similar method as in the first and second case to extract the following demonstration. In Fig. 3, the magnetic flux density of the particle is shown when it is assumed under the action of the Lorentz force $\phi\left(\mathbf{b}_{\mathbf{q}}\right)$. 


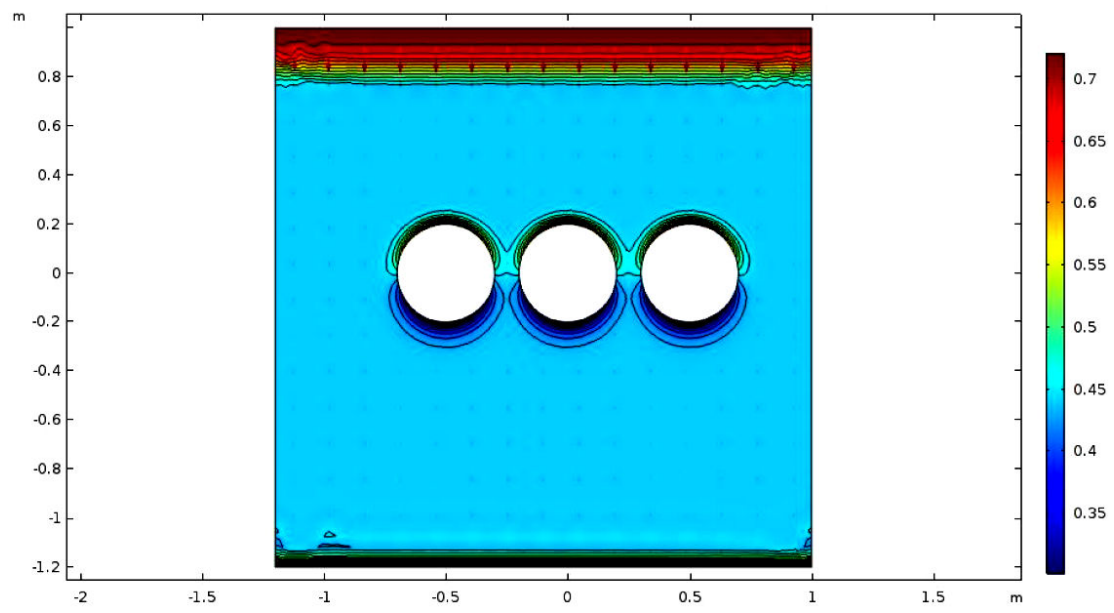

FIGURE 3 . The magnetic flux density with Lorentz force $\phi\left(\mathbf{b}_{\mathbf{q}}\right)$.

\section{Conclusion}

Flows perform an essential part in geometric design and style, applied physics, and structural motion. In ths paper, we have investigated a different approach by considering the directional flows of the velocity magnetic particles and the quasi frame. We have provided several different characterizations of curvatures regarding plenty of differential equations in space. These characterizations may further be used to in- vestigate the directional flows of magnetic particles. Another purpose of future studies will be to explore the unified formulations of the systems composed of arbitrary dyons, magnetic and electric charges of manifolds with magnetic flux lines. The magnetohydrodynamic model of the magnetic surfaces reduced from the magnetic flux surfaces of distinct types will also be the subject of another project. This project eventually aims to investigate significant features of the flux maximizing and minimizing flows.
1. A.M Smith, Polarization and magnetooptic properties of singlemode optical fiber, Appl. Opt. 17 (1978) 52. https://doi. org/10.1364/AO.17.000052

2. J.N. Ross, The rotation of the polarization in low birefringence monomode optical fibres due to geometric effects, Opt. Quantum Electron. 16 (1984) 455.https : / doi.org/10. $1007 / \mathrm{BF} 00619638$

3. A. Tomita and Y. Chiao, Observation of Berry's topological phase by use of an optical fiber, Phys. Rev. Lett. 57 (1986) 937. https://doi.org/10.1103/ PhysRevLett.57.937

4. R.Y. Chiao and Y.S. Wu, Manifestations of Berry's topological phase for the photon, Phys. Rev. Lett. 57 (1986) 933. https : //doi.org/10.1103/PhysRevLett.57.933

5. F.D.M Haldane, Path dependence of the geometric rotation of polarization in optical fibers, Opt. Lett., 11 (1986) 730. https://doi.org/10.1364/OL.11.000730

6. T. Körpınar and R.C. Demirkol, Electromagnetic curves of the linearly polarized light wave along an optical fiber in a 3D semiRiemannian manifold, J. Mod. Opt., 66 (2019) 857. https: //doi.org/10.1080/09500340.2019.1579930

7. T Körpınar, R.C. Demirkol and Z. Körpınar, Soliton propagation of electromagnetic field vectors of polarized light ray traveling in a coiled optical fiber in Minkowski space with
Bishop equations, Eur. Phys. J. D 73 (2019) 203. https: //doi.org/10.1140/epjd/e2019-100250-7

8. T. Körpınar, R.C. Demirkol and Z. Körpınar, Soliton propagation of electromagnetic field vectors of polarized light ray traveling in a coiled optical fiber in the ordinary space, Int. J. Geom. Methods Mod. Phys. 16 (2019) 1950117. https: //doi.org/10.1142/S0219887819501172

9. T. Körpınar, R.C. Demirkol and Z. Körpınar, Soliton propagation of electromagnetic field vectors of polarized light ray traveling along with coiled optical fiber on the unit 2-sphere $\mathbb{S}^{2}$., Rev. Mex. Fís. 65 (2019) 626-633. https : // doi.org/10. 31349 /revmexfis.65.626

10. T. Körpınar and R.C. Demirkol, Electromagnetic curves of the linearly polarized light wave along an optical fiber in a 3D Riemannian manifold with Bishop equations, $O p$ tik, 200 (2020) 163334.https://doi.org/10.1016/j. ijleo.2019.163334

11. G.L. Lamb, Solitons on moving space curves, J. Math. Phys., 18 (1977) 1654. https://doi.org/10.1063/1. 523453

12. S. Murugesh and R. Balakrishnan, New connections between moving curves and soliton equations, Phys. Lett. A, 290 (2001) 81. https://doi.org/10.1016/S0375-9601(01) 00632-6 
13. M.V. Berrry, Quantal phase factors accompanying adiabatic changes. Proceedings of the Royal Society of London. A. Proc. R. Soc. Lond. A, 392 (1984) 45. https://doi.org/10. $1098 /$ rspa.1984.0023

14. X.S. Fang and Z.Q. Lin, Field in single-mode helicallywound optical fibers, IEEE Trans. Microw. Theory Techn. 33 (1985) 1150. https://doi.org/10.1109/TMTT. 1985.1133187

15. J.N. Ross, The rotation of the polarization in low birefringence monomode optical fibres due to geometric effects, Opt. Quantum Electron. 16 (1984) 455.https : / / doi .org/10. $1007 / \mathrm{BF} 00619638$

16. L. Lu, J. D. Joannopoulos, and M. Soljačić, Topological photonics, Nat. Photonics 8 (2014) 821, https://doi.org/ $10.1038 /$ nphoton.2014.248

17. F. Wassmann and A. Ankiewicz, Berry's phase analysis of polarization rotation in helicoidal fibers, Appl. Opt. 37 (1998) 3902. https://doi.org/10.1364/AO.37.003902

18. R. Balakrishnan, R. Bishop and R. Dandoloff, Geometric phase in the classical continuous antiferromagnetic Heisenberg spin chain, Phys. Rev. Lett. 64 (1990) 2107. https : / / doi . org/ $10.1103 /$ PhysRevLett . 64.2107
19. R. Balakrishnan, R. Bishop and R. Dandoloff, Anholonomy of a moving space curve and applications to classical magnetic chains, Phys. Rev. B, 47 (1993) 3108. https : //doi.org/ 10.1103/PhysRevB.47.3108

20. R Balakrishnan and R Dandoloff, The Schrödinger equation as a moving curve, Phys. Lett. A, 260 (1999) 62-67. https : //doi.org/10.1016/S0375-9601(99)00492-2

21. J. Anandan, The geometric phase, Nature. 360 (1992) 307. https://doi.org/10.1038/360307a0

22. T.Körpinar and R.C. Demirkol, Gravitational magnetic curves on 3D Riemannian manifolds, Int. J. Geom. Methods Mod. Phys. 15 (2018) 1850184. https: //doi.org/10.1142/ S0219887818501840

23. T. Körpinar and R.C. Demirkol, Frictional magnetic curves in 3D Riemannian manifolds, Int. J. Geom. Methods Mod. Phys. 15 (2018) 1850020. https://doi.org/10.1142/ S0219887818500202

24. M.P. Carmo, Differential Geometry of Curves and Surfaces, (Prentice-Hall, New Jersey, 1976).

25. M. Dede, C. Ekici and H. Tozak, Directional tubular surfaces, Int. J. Algebra, 9 (2015) 527. http://dx.doi.org/10. $12988 /$ ija.2015.51274 